\title{
Computational techniques and validation of 3-dimensional viscous/turbulent codes for internal flows*
}

\author{
B LAKSHMINARAYANA, K R KIRTLEY and M WARFIELD
}

Aerospace Engineering Department and Applied Research Laboratory, The Pennsylvania State University, University Park, PA 16802, USA

MS received 12 April 1989

\begin{abstract}
Computational techniques and codes developed for the prediction of three-dimensional turbulent flows in internal configurations and rotor passages are described. Detailed calibration and validation of the flow fields in $90^{\circ}$ curved ducts, cascades, end-wall flows and turbomachinery rotors are presented. Interpretation and comments on accuracy, level of agreement with various turbulence models and limitations of the codes are described. The single pass space-marching code is found to be efficient for curved duct and two-dimensional cascade flows. Multipass space-marching, time-marching and zonal methods are found to be accurate for complex situations. The efficiency and accuracy of a zonal technique, with considerable saving in computational time, is demonstrated.
\end{abstract}

Keywords. Validation of codes; internal flows; computational flows; 3-D turbulent flows.

\section{Introduction}

Internal flows, such as the flow through straight and curved ducts, cascades, diffusors, nozzles, combustion chambers, turbomachinery stators and rotors are among the most complex flows encountered in practice. They are almost invariably threedimensional and turbulent and are very frequently unsteady. In many instances (e.g., turbomachinery cascade and rotor), boundary conditions which are difficult to enforce are encountered. In addition, complex strain is encountered due to threedimensionality, curvature and rotation effects. The governing equations are elliptic, parabolic, or hyperbolic depending on the flow variables and regions under consideration. It is not unusual to have regions where all three equation types are valid in the same passage. The system of equations governing this flow requires expensive iterative solution procedures which may become prohibitively expensive for application to full turbomachinery blade rows or coupled systems consisting of ducts, rotors, stators, etc.

\footnotetext{
* Paper presented at AGARD Symposium "Validation of Computational Fluid Dynamics," May 2-5, 1988, Lisbon, Portugal
} 
Substantial progress has been made in resolving viscous internal flow through simple configurations or approximate flow in complex configurations. McNally \& Sockol (1981) and AGARD (1985) provide good reviews on these techniques. The next decade will see a major thrust in the development of efficient and accurate solution of multi-row turbomachinery (e.g., multi-stage) with three-dimensional turbulent flow and heat transfer.

The two levels of code verification are code calibration and code validation. Code calibration is carried out with some of the simplest flows and geometry for which exact analytical solutions or benchmark quality data are available. Examples of candidates for calibration are the laminar flow in a duct, cavity, or flow over a flat plate. This will enable decoupling of errors associated with the technique and the code from the inaccuracy of turbulence models, grids etc. The next step is the calibration for turbulence models, grids etc., where well-documented turbulence data (e.g., turbulent flow on a flat plate with and without pressure gradient) is compared with the computation.

The code developer is now ready for the next level of check; namely, validation. This is carried out in more complex configurations that are representative of practical geometries. Examples of these are the flow through cascades of blades, curved ducts (convex and concave, S-duct etc.), transonic flows over an airfoil, wing body combinations etc., for which accurate sets of data are available. The last stage is the prediction. This may include such state-of-the-art configurations as rotors (helicopter and turbomachinery), aircraft or components.

As described earlier, the major objective of this research is to develop original, efficient and accurate computational techniques and, most importantly, to calibrate and validate these codes as well as provide predictions for complex flows.

A need exists for efficient solution of the incompressible Navier-Stokes equations. Large classes of industrial, aerospace, marine and land flow devices have incompressible or nearly incompressible flow. The present effort has concentrated mainly on the computation of incompressible, turbulent and three-dimensional flows in internal flow passages, including turbomachinery.

\section{Governing equations and turbulence models}

The governing equations for incompressible and rotating flows written in vector form are

$$
\begin{aligned}
& S_{m} \frac{\partial q}{\partial t}+\frac{\partial E}{\partial x}+\frac{\partial F}{\partial y}+\frac{\partial G}{\partial z}-\frac{1}{\operatorname{Re}}\left(\nabla^{2} q\right)=S, \\
& q=\left[\begin{array}{c}
0 \\
u \\
v \\
w
\end{array}\right], \quad E=\left[\begin{array}{c}
u \\
u^{2}+p \\
u v \\
u w
\end{array}\right], \quad F=\left[\begin{array}{c}
v \\
u v \\
v^{2}+p \\
v w
\end{array}\right], \quad G=\left[\begin{array}{c}
w \\
u w \\
v w \\
w^{2}+p
\end{array}\right], \\
& S=\left[\begin{array}{c}
0 \\
B_{1} \\
B_{2} \\
B_{3}
\end{array}\right], \quad B_{i}=\left[-2 \varepsilon_{i j k} \Omega_{j} \Omega_{k}-\Omega_{m} x_{m} \Omega_{i}+\Omega_{n} \Omega_{n} x_{i}-\overline{\partial u_{i}^{\prime} u_{j}^{\prime}} / \partial x_{j}\right], \\
& S_{m}=\operatorname{diag}(0,1,1,1),
\end{aligned}
$$


where $u, v, w$ are velocities in the $x, y, z$ directions, respectively ( $x$ being the dominant flow direction), $i=1,2$, and 3 represent $x, y, z$ momentum equations, $j, k, m, n$ are dummy indices. The Einstein summation convention is utilized. Coriolis force, centrifugal force and turbulence stresses are included in (1). For example, if the rotation is about the $x$ axis $\left(\Omega_{2}=\Omega_{3}=0\right)$,

$$
\begin{aligned}
& B_{1}=0-\overline{\partial u_{1}^{\prime} u_{j}^{\prime}} / \partial x_{j}, \quad B_{2}=\Omega^{2} y+2 \Omega w-\overline{\partial u_{2}^{\prime} u_{j}^{\prime}} / \partial x_{j}, \\
& B_{3}=\Omega^{2} z-2 \Omega v-\overline{\hat{\gamma} u_{3}^{\prime} u_{j}^{\prime}} / \partial x_{j} .
\end{aligned}
$$

If $y(j=2)$ is the boundary layer coordinate, then $j=2, i=1,2$, and 3 are the only dominant shear stress and intensity terms in these equations.

Equation ( 1 ) is hyperbolic in time and can be solved using a time-marching algorithm. The Navier-Stokes equations can be simplified using realistic assumptions. For example, if the streamwise, pressure gradient $(\partial p / \partial x)$ and viscous diffusion $\left(u_{x x}\right)$ terms are small, they may be neglected, and the resulting system is termed the parabolized Navier-Stokes equations (PNS). If the $u_{x x}$ is dropped and streamwise pressure gradient is treated elliptically, then the equations become the partially parabolized Navier-Stokes equations (PPNS). The simplified forms of the Navier-Stokes equations are increasingly more efficient as terms are dropped; PNS requires more than one order of magnitude less computation time and less computer storage than the full Navier-Stokes equations.

The techniques developed (or used) at Penn State can be generally classified as the single pass space-marching techniques, pseudocompressibility multipass spacemarching technique, pseudocompressibility time-marching technique, and the zonal technique, which is a combination of the first and the last techniques. As some of the boundary conditions and turbulence modelling are common for all these codes, they will be described first. Only the turbulent flow will be covered in this paper. In all the space-marching techniques, the steady flow is resolved neglecting time derivative in (1).

\subsection{Turbulence models}

Internal flows cover a wide spectrum of geometries, starting from a simple duct to the complex three-dimensional rotor. It is neither desirable nor efficient to use the same model for all the flows. For example, in two-dimensional flows with a mild pressure gradient, both the algebraic eddy viscosity and the two-equation model $(k-\varepsilon)$ provide good predictions. In three-dimensional flows with curvature and rotation, it is necessary to resort to more complex and non-isotropic models such as Algebraic Reynolds Stress Models (ARSM). A review of various models available and their suitability to various situations is given in Lakshminarayana (1986). All the lower and higher levels of turbulence models are incorporated in these codes. The Algebraic Eddy Viscosity Model (AEVM-BL) used by Baldwin \& Lomax (1978) is incorporated, as is the $k-\varepsilon$ model used by Jones \& Launder (1973). In both these models the shear stress is represented by

$$
-\overline{u_{i}^{\prime} u_{j}^{\prime}}=v_{t}\left(\frac{\partial u_{i}}{\partial x_{j}}+\frac{\partial u_{j}}{\partial x_{i}}\right)-\frac{2}{3} \delta_{i j} k .
$$

In the algebraic eddy viscosity model, $v_{t}$ is an empirical expression, while in the $k-\varepsilon$ 
model it is related to the local $k$ and $\varepsilon$ given by $v_{t}=c_{\mu} k^{2} / \varepsilon$.

The Boussinesq assumption implies that the Reynolds stress tensor is aligned with the mean strain rate. Isotropic assumption implies that $v_{t}$ is independent of the direction. Here, a single value of $v_{t}$ is used to relate all the shear stresses. The Reynolds stress and algebraic stress models rectify this deficiency and allow for more physics to be incorporated into the model. However, the solution of the full Reynolds stress equations is complex; hence the ARSM becomes an efficient alternative. The ARSM equates the source terms in modelled Reynolds stress equations to the corresponding source terms in the turbulence kinetic energy equation (which implies that the ratio of the transport of Reynolds stress to the transport of turbulence kinetic energy is equal to $\overline{u_{i}^{\prime} u_{j}^{\prime}} / k$ ). This model was first proposed by Rodi (1976) for stationary flows, but was later extended by Galmes \& Lakshminarayana (1984) to rotating flows. Warfield \& Lakshminarayana (1987) manipulated these equations to derive values of $c_{\mu}$ which include the effect of three-dimensionality and rotation. Their equations are given by

$$
\begin{aligned}
& -\overline{u^{\prime} v^{\prime}}=c_{\mu 1} \frac{k^{2}}{\varepsilon} \frac{\partial u}{\partial y}, \quad-\overline{u^{\prime} w^{\prime}}=c_{\mu 2} \frac{k^{2}}{\varepsilon} \frac{\partial u}{\partial z}, \\
& -\overline{v^{\prime} w^{\prime}}=c_{\mu 3} \frac{k^{2}}{\varepsilon} \frac{\partial v}{\partial z}+c_{\mu 4} \frac{k^{2}}{\varepsilon} \frac{\partial w}{\partial y},
\end{aligned}
$$

where

$$
c_{\mu i}=f_{i}\left(\frac{P}{\varepsilon}, \frac{k \Omega_{k}}{\varepsilon}, \frac{\partial u_{i} / \partial x_{J}}{\Omega_{k}}, \frac{\partial u_{i} / \partial x_{J}}{\partial u_{k} / \partial x_{m}}\right), \quad i=1,2,3,4 .
$$

Thus $c_{\mu}$ is a non-isotropic property dependent on the local flow field, field, rotation, production and dissipation; $f_{i}$ is given in Warfield \& Lakshminarayana (1987). Hence $k-\varepsilon$ transport equations with a variable $c_{\mu}$ effectively incorporate many of the complex strain effects caused by the three-dimensionality and the rotation. In the calculation of vectorial $c_{\mu}$ in the current time or spatial step through the ARSM, the production terms are derived from the previous time or spatial step to avoid nonlinearity in the algebraic turbulence stress equations.

\subsection{Boundary conditions}

There are common elements in the boundary conditions employed in both the timemarching and space-marching techniques, but the implementation differs for internal flow problems. Four boundary conditions are considered.

(i) In surface boundaries, the velocities and the normal pressure gradient are zero. In many applications, especially a rotor, the grid resolution and thin boundary layers encountered make it desirable to use a turbulent slip velocity. The slip velocity is based on the law of wall given by $\partial u / \partial y=u^{*} / \kappa y$, where $u^{*}$ is the friction velocity, and $\kappa$ is the Von Karman constant. In space-marching, a two-point backward differencing is used for the pressure gradient condition in order to couple the odd-even points.

(ii) Inlet conditions are generated from the available experimental data.

(iii) Downstream boundary conditions differ depending on the technique used. For example, in single pass space-marching, no downstream boundary condition is 
specified. In pseudocompressibility multipass techniques, only pressure is used as a boundary condition. In pseudocompressibility time-marching, the pressure and velocity components were extrapolated while conserving the mass flow.

(iv) In cascades and turbomachinery rotors, periodicity has to be enforced. In the time-marching code, the periodicity is enforced through the use of an implicit periodic matrix solver. In the pseudocompressibility space-marching technique, periodic conditions are applied to all variables in an explicit manner. Periodic nodes are treated as internal points, and averaging is used during the iterative matrix inversion process in the cross plane. Periodic conditions are applied to all variables $u, v, w, p, k, \varepsilon, c_{\mu i}$ etc.

(v) In ducts with symmetrical inlet flow, the symmetry conditions have to be enforced. The gradients of pressure and streamwise velocity component in the transverse direction are set to zero. A two-point backward difference scheme is employed for these gradients both implicitly and explicitly.

In the case of the compressor rotor, the annulus wall was modelled as a moving wall (relative to the rotor) without the tip clearance, with the tangential velocity equal but opposite to the blade tip speed. The intention here is to capture the flow accurately away from the annulus walls on the blade surface, without resolving the tip flows.

\subsection{Transformation of equations and grid generation}

For complex geometries, it is desirable to solve the governing equations on a generalized coordinate system. The following transformation is used to recast (1) in a body-fitted coordinate; $\xi=\xi(x, y, z), \eta=\eta(x, y, z), \zeta=\zeta(x, y, z)$.

The cartesian velocity components and the pressure are still dependent variables. However, the equation is now solved on a generalized coordinate system. The transformed equations in a rotating coordinate system are given in the appendix of Govindan \& Lakshminarayana (1988).

An algebraic grid generation technique is used since this technique is simple and efficient, especially for 3-D configurations. The grid generation technique for a turbomachinery rotor will be briefly described, and those for curved ducts, S-ducts and cascades are simple derivatives of the generalized case. The geometry of the blade passage, hub, annulus wall and blade profile is input to the cylindrical coordinate system $(r, \theta, z)$. First, a two-dimensional grid in the $z-r$ plane is established giving cylindrical sheets of which two-dimensional $\mathrm{H}$-grids in the blade to blade direction ( $\theta z$ plane) are established to follow the blade profiles through interpolation. Special care is taken to obtain a smooth grid which is periodic in $\theta$. The $\mathrm{H}$-grid is well-suited for ducts, cascades, rotors or other configurations with sharp leading and trailing edges.

\section{Computational techniques}

As indicated earlier, it is inefficient to solve the full Navier-Stokes equations, and inaccurate to solve only the parabolized equation for some turbomachinery and internal flows. Hence, there is need to develop techniques to capture the essential physics efficiently. With this objective in mind, the turbomachinery group at Penn 
State has developed several techniques and codes for prediction of internal flows. These techniques will be described briefly, and the reader is referred to cited theses and papers for details. The main emphasis of this paper will be in validation, commenting on the accuracy of the grid, turbulence mode!, technique, and the effect of boundary conditions. The techniques and codes can be classified as follows:

(i) single pass space-marching technique (SPSALT);

(ii) space-marching technique with a pressure solver (SALTPS);

(iii) multipass pseudocompressibility space-marching technique (MPSALT);

(iv) pseudocompressibility time-marching technique (INS3DPSU);

(v) zonal technique (hybrid scheme incorporating techniques $i$ and iv, zonal 3-D).

The abbreviations used for these codes are shown in parentheses above. For example, SPSALT stands for single pass space-marching algorithm for laminar and turbulent flows, INS3DPSU is a code developed by Kwak et al (1986) at the NASA Ames Research Center and modified (Warfield \& Lakshminarayana 1987) by Penn State University (PSU) to include a $k-\varepsilon /$ ARSM turbulence model, convergence accelerators, and periodic boundary conditions for $\mathrm{H}$-grids.

In many internal flows of practical applications (e.g. curved ducts, cascades, turbomachinery passages), a dominant flow direction can be identified and these flows can be predicted by marching along the dominant flow direction. In most subsonic flows, the streamwise pressure gradient is important, even though the streamwise diffusion term is small for thin unseparated shear layers. Many techniques have been developed to include the elliptic effects caused by these pressure gradients. No attempt will be made here to review the techniques developed by various investigations, as detailed reviews can be found in Rubin (1980) and Kirtley (1987). The problems of properly satisfying local continuity in the entirc flow field through the iterations remain in the parabolic methods. A further drawback of some of these techniques is separation of the vector governing equation into individual scalar equations during the solution and use of an iterative scheme to couple the equations. In a flow field with strong secondary flow, the strong coupling between the pressure field and the transverse velocity field would slow the convergence. All the techniques and codes described in this paper solve these equations simultaneously; the methods differ in the handling of pressure effects. These are described below. The time derivative terms of (1) are dropped in all the space-marching techniques.

Govindan \& Lakshminarayana (1988) developed a space-marching technique to compute 3-D viscous flow fields. The Navier-Stokes equations (1) have been posed as an initial value problem by neglecting the streamwise diffusion term $u_{x x}$ and treating the streamwise pressure gradient in the $E$ vector of (1) as a known source term. A noniterative linear block implicit (LBI) scheme from Briley \& McDonald (1980) is used to solve the equations. Initially, the pressure is specified from an inviscid code. To conserve the global mass flow in computations, the mean streamwise pressure gradient is computed from the predicted velocity field and the corrected streamwise pressure gradient is computed for use at the next streamwise station. Since most of the results from this code are published (Govindan \& Lakshminarayana 1988; Gorski et al 1985), these results will not be presented here. The reader is referred to Gorski et al $\cdot(1985)$ for accuracy of turbulence models ( $k-\varepsilon$, versus algebraic eddy viscosity), slip versus no-slip conditions, error due to grid resolution etc. 


\subsection{Single pass space-marching code (SPSALT)}

The earlier technique (Govindan \& Lakshminarayana 1988) was developed for compressible flow, including the energy equation. The method was very sensitive to the specified pressure gradient, and difficulties were encountered at very low Mach numbers. This motivated the development of a new technique (Pouagare \& Lakshminarayana 1986) specifically for incompressible flows. An cigenvalue analysis of (1) (two-dimensional form) for marching in the streamwise direction shuws that two of the eigenvalues are imaginary. Such a situation precludes the use of the single sweep space-marching method without uncoupling the pressure. Therefore the streamwise pressure was split into implicit and explicit parts, so that the vector $E$ was split as follows:

$$
\begin{aligned}
& E=E_{\mathrm{im}}+E_{\mathrm{ex}} \\
& E_{\mathrm{im}}=\left[\begin{array}{c}
u \\
u^{2}-|\sigma| p \\
u v \\
u w
\end{array}\right], \quad E_{\mathrm{ex}}=\left[\begin{array}{c}
0 \\
|\sigma| p+p \\
0 \\
0
\end{array}\right] .
\end{aligned}
$$

Substitution of (2) in (1) makes the equation hyperbolic in space [neglecting the time derivative in (1)], thus enabling a streamwise integration. Pouagare \& Lakshminarayana (1986) show that $\sigma$ should be small and negative; the introduction of $|\sigma|$ changes the character of the equation from elliptic to hyperbolic. The numerical algorithm used is the same as that used earlier (Govindan \& Lakshminarayana 1988). This technique is very efficient and accurate provided the prescription of $\partial p / \partial x$ is accurate. In most situations in internal flows, cascades, and turbomachinery, the pressure distribution can be accurately specitied (as it is mostly an inviscid phenomena) through the use of an efficient panel method code. Validation of this code was carried out for laminar and turbulent flows in a curved duct, cascade blade boundary layer and cascade end-wall flows. Results from this code will be described later.

\subsection{Space-marching technique with a pressure-solver (SALTPS)}

The sPSALT code was run without success in a multipass mode. Kirtley (1987) has shown that the method is not stable in a multipass mode. If the prescribed or assumed pressure is not accurate, the calculated pressure may deviate considerably from the actual pressure. The ellipitic effects are not captured, and the technique is therefore unstable. Hence, a Poisson solver for pressure was developed to capture the elliptic effects. For example, for two-dimensional flows, the pressure is given by

$$
\nabla^{2} p=2\left[\frac{\partial u}{\partial x} \frac{\partial v}{\partial y}-\frac{\partial u}{\partial y} \frac{\partial v}{\partial x}\right]
$$

This equation was solved using a standard line relaxation technique. No convergence accelerators were included. Details of this code are given in Kirtley (1985).

It should be emphasized here that even though a Poisson solver is used to correct the pressure, the technique solves all the equations (continuity and momentum) 
simultaneously and thus differs from the earlier parabolic marching techniques in which the equations are uncoupled and solved consecutively. The convergence, especially for flows with large pressure gradients, is likely to be slow in an uncoupled system. The transformed equations are marched in the $\xi$ (streamwise) direction from the initial line using an assumed initial pressure field. For cascade flows, the inviscid pressure field was calculated from a panel code. After the domain was swept once, the computed velocity field was used in (3), which was iterated to convergence. This new pressure is then used again in (1) to update the velocity field. It was found that the change in the velocity field due to the updated pressure was very small. The velocity field was reasonably accurate after the first pass (from a prescribed accurate inviscid pressure), but the pressure had to be iterated to obtain accurate viscous corrections.

The salTPS code was used mainly for two-dimensional viscous flow calculation. An attempt was made to develop a three-dimensional pressure solver, but the convergence was very slow; hence a new and more efficient technique described below was developed for 3-D viscous flow. This is designated as a multipass space-marching technique which utilizes the pseudocompressibility technique and involves no separate pressure solver.

\subsection{Multipass space-marching method (MPSALT)}

In earlier parabolic-marching methods (Rubin 1980), the continuity equation is satisfied only through an iterative process, and not directly. When the continuity and the momentum equations are solved in a coupled fashion, as in the methods described above, forward differencing of the streamwise pressure is sufficient to achieve a stable marching. Kirtley (1987) has indicated that the pressure gradients cannot be relaxed in order to achieve a stable multipass procedure without the use of auxiliary equations (e.g. a Poisson equation). Since the continuity equation is coupled to the momentum equation, some conditions must be relaxed during computation in order to solve the complex flows. The only condition left to be relaxed in a practical way is the continuity equation. The method of pseudocompressibility developed by Chorin (1967) is incorporated to yield a marching technique which is stable and convergent in a global iteration for complex geometries. A complete description of this technique is given in Kirtley (1987); a brief description follows.

Chorin's (1967) method, developed for incompressible flow, relaxes the continuity equation while keeping the equation coupled to the momentum equations. In this method, an artificial time derivative is added to the continuity equation as follows,

$$
(1 / \beta)(\partial p / \partial t)+\nabla \cdot V=0
$$

where $\mathbf{V}$ is the total velocity vector. This relaxes the continuity constraint during a time-marching integration of the Navier-Stokes equation. At convergence, the computed flow field is divergence-free and hence represents steady flow solution. Since it is a space-marching technique that is under consideration here, and no time derivatives are present, the continuity equation is written as

$$
a\left(p^{n}-p^{n-1}\right)+\nabla \cdot V=0
$$

where $n$ is a global iteration and $a$ is analogous to $(1 / \beta) \Delta t$ in Chorin's (1967) method. Successive passes of the domain may be thought of as advancement in time. Substitution of (5) and (1) with $\left(S_{m}=0\right)$ results in the following changes in vectors 
$E$ and $F$.

$$
E=\left[\begin{array}{c}
a p+u \\
u^{2}+p \\
u v \\
u w
\end{array}\right], \quad S=\left[\begin{array}{c}
a \partial_{x} p^{n}-p_{i}^{n}+p_{i}^{n-1} \\
B_{1} \\
B_{2} \\
B_{3}
\end{array}\right]
$$

Vectors $q, F, G$ are the same as in (1). An eigenvalue analysis (Kirtley 1987), as well as global stability analysis, has shown that forward marching with (1) and (4) is a stable solution technique and that the method will be convergent in a multipass mode.

Equation (1) with (5) was solved using the following iterative LBI scheme to reduce the effect of the splitting error in the computation. This error, unlike that for time marching methods, was multiplied by the streamwise change in the dependent vector $\Delta q$ which does not tend to zero.

$$
\left(L_{\xi^{\bullet}}+L_{\eta} \bullet+L_{\xi}\right)\left(\Delta q^{n}-\Delta q^{n-1}\right)=R-\left(L_{\xi \bullet}+L_{\eta} \bullet+L_{\zeta} \bullet \Delta q^{n-1}=R^{\prime} .\right.
$$

$L_{\xi}$ is the convection term in the marching direction $(\xi)$ in the body-fitted coordinate system; $L_{\eta}$ and $L_{\zeta}$ are the convection plus the diffusion terms in the transverse directions $(\eta, \zeta)$ in the body-fitted coordinate system. The following equations were solved.

$$
\begin{aligned}
& \left(L_{\xi \bullet}+L_{\eta}\right) \Delta s^{*}=R^{\prime}, \\
& \left(L_{\xi \bullet}+L_{\zeta}\right) \Delta s^{n}=L_{\xi} \Delta s^{*},
\end{aligned}
$$

where $\Delta s^{n}=\Delta q^{n}-\Delta q^{n-1}$. The convergence is improved by including a relaxation parameter,

$$
\Delta q^{n}=\Delta q^{n-1}+\omega \Delta s^{n} .
$$

Convergence of two orders of magnitude is generally achieved with $\omega=0 \cdot 8$. Once convergence is achieved, the solution is advanced to the next streamwise station. Once the complete domain has been swept, the new pressure field is stored and used in the vectors for repeated passes of the domain. The global iterative procedure is terminated when the computed pressure no longer changes with iteration. The algorithm has been calibrated (Kirtley 1987) for well-known laminar solutions, and the code is used to validate the turbulent data in an S-duct, a curved $90^{\circ}$ bend, cascade boundary blade layers and wakes, end-wall flow and compressor rotor passage flow.

\subsection{Pseudocompressibility time marching code (INS3DPSU)}

As mentioned earlier, this technique involves modifying the elliptic equations governing incompressible flows to hyperbolic equations by the introduction of a time derivative in the continuity equation (4). Substitution of (4) in (1) results in Chorin's formulation,

$$
\begin{aligned}
& q=(p / \beta, u, v, w), \quad S_{m}=\operatorname{diag}(1,0,0,0), \\
& S=\left[(1 / \beta)\left(\partial p^{n-1} / \partial t\right), B_{1}, B_{2}, B_{3}\right] .
\end{aligned}
$$

This introduces pressure waves of finite speed through the pseudocompressibility parameter $\beta$. Kwak et al (1986) utilized this technique and developed a code for threedimensional viscous flow. Kwak et al (1986) discussed the stability criteria for $\beta$, and 
suggested lowe: and uppei bounds for $\beta$. The code, designated INs3D by Kwak et al (1986), has been modified by the Penn State group to include the periodic boundary conditions for $\mathrm{H}$-grids and convergence acceleration via a variable time step determined from a local CFL condition. Their equations and code were modified to include vector $S$ (rotation effects) and the turbulence modelling in (1). These modifications are described by Warfield (1987). The code was utilized to predict the Tow field in cascade biades, end-walls and, most importantly, a compressor rotor. The solution technique used by Kwak et al (1986) for the numerical integration of (1) is the standard Euler implicit approximate factorization scheme, which reduces the threedimensional equations to a form amenable to solution by block tridiagonal inversion methods. Both explicit and implicit smoothing were utilized to facilitate stability and convergence of the solution. The codz has been validated against data available for internal flows.

\subsection{Zonal technique (zonal 3-D)}

Complete solution of the full Navier-Stokes equation in all regions of flow is inefficient, expensive, and often unnecessary. For example, high Reynolds number viscous flows require resolution of thin viscous layers and an inviscid core. This situation often requires a large number of grid points in the viscous layer, which may not be possible with a Navier-Stokes method, due to storage and run-time limitations. Parabolized equations applied to the viscous layers, and Euler equations applied to ihe inviscid core, may result in a substantial decrease in computational time and storage. Even though the Navier-Stokes equations are universally valid, reduced forms are more appropriate and efficient in some rcgions. For example, many duct llows are parabolic or weakly elliptic, and downstream boundary conditions are difficult to apply. Many complex flows contain regions with natural elliptic and parabolic character. Flow over an aircraft, flow through a turbomachinery blade row and wakes are examples of such flows. Consider a turbomachinery blade row with an upstream annulus and downstream annulus. The parabolic solutions are appropriate upstream (say $1 / 3$ to 1 chord) and downstream (including wake). The passage inside contains an inviscid region where the complete Euler equation is needed, and thin layers can be handled through parabolic or space-marching methods. This has motivated development of a zonal equation method. Warfield (1987) has provided a critical review of these techniques. A schematic of the zonal technique developed (Warfield \& Lakshminarayana 1987) is shown in figure 1. The zonal equation method described in this paper involves solving the full Navier-Stokes equation in the elliptic zone using the INS3DPSU code described earlier, and the parabolized Navier-Stokes equation using SPSALT in the parabolic zone, with a buffer zone where ellipticity due to $u_{x x}$ and $p_{x}$ is changing from nearly zero to $0(1)$ as an interactive boundary. The technique development and validation is described in Warfield \& Lakshminarayana (1987) and Warfield (1987)) and the application is explained in this paper.

The quantification of ellipticity defined through parameters $\varepsilon_{1}$ and $\varepsilon_{2}$, is achieved before implementation of the zonal technique. For example, the ellipticity for a duct flow can be characterized as follows,

$$
\varepsilon_{1}=\left[(\partial p / \partial y) u_{x x}^{2}\right] /\left[(\partial p / \partial x) u_{y y}^{2}\right], \quad \varepsilon_{2}=\left[(\partial p / \partial z) u_{x x}^{2}\right] /\left[(\partial p / \partial x) u_{z z}^{2}\right]
$$



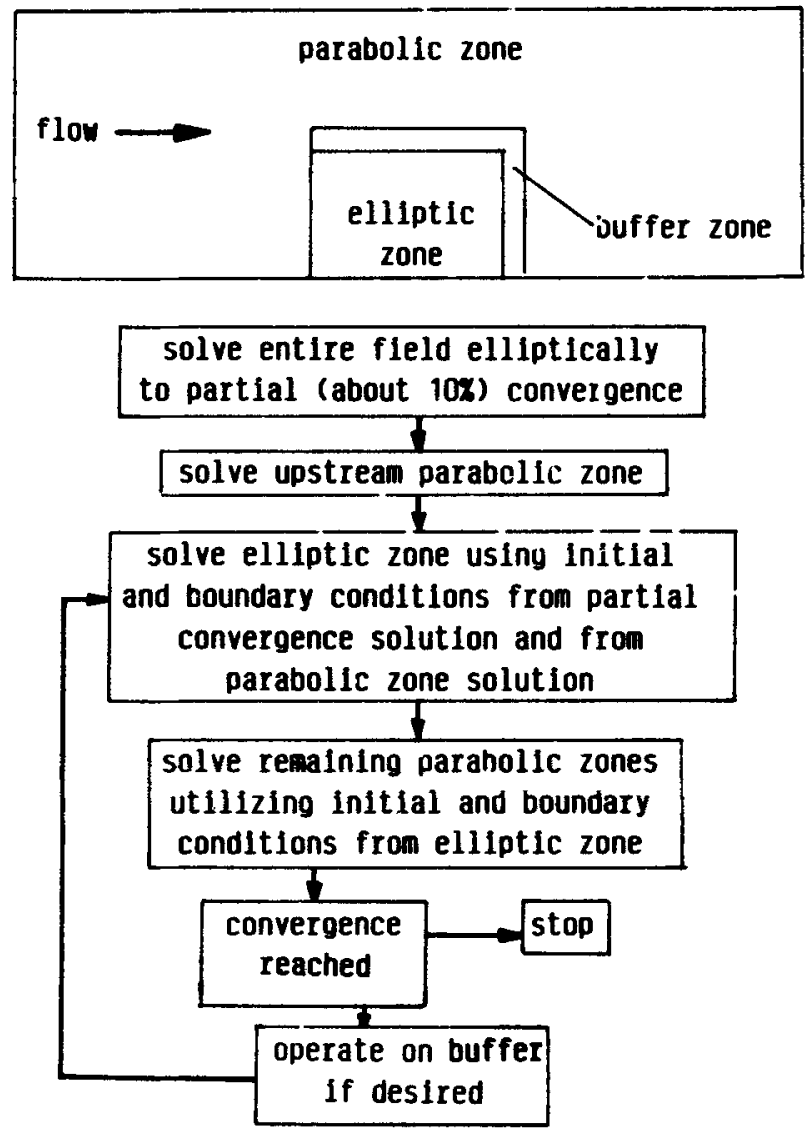

Figure 1. The zonal equation method algorithm.

$\varepsilon_{1}$ and $\varepsilon_{2}$ go to zero in a purely parabolic flow, since $\partial p / \partial y=\partial p / \partial z=u_{x x}=0$ for fully developed flow and $u_{y y}, u_{z z}$, and $\partial p / \partial x$ (which drives the flow) are non-zero. For more complex situations, such as those dominated by inviscid flow, one may need different characterization. Here $\varepsilon_{1}$ characterizes the ellipticity in the $x$ direction, and $\varepsilon_{2}$ represents ellipticity in the $z$ direction, the $y$ direction being the boundary layer coordinate.

The elliptic system is partially converged (approximately 10\% computation time) as shown in figure 1 to derive an initial assumed pressure field for a single pass space-marching technique and to establish the elliptic and parabolic zones. The parameters $\varepsilon_{1}$ and $\varepsilon_{2}$ are used to establish boundaries. Since the single pass technique is sensitive to the assumed pressure field, it is very important to transmit the pressure information from the elliptic zone to the parabolic zone via a buffer zone. The pressure field is continually updated in the buffer zone and, if necessary, the parabolic zone is swept again (it then becomes a multipass) as shown in figure 1. The zonal 3-D code was calibrated for a post and a bump in a duct; this is described in Warfield \& Lakshminarayana (1987) and Warfield (1987). Validation wth experimental data in a compressor rotor is described in the next section. 


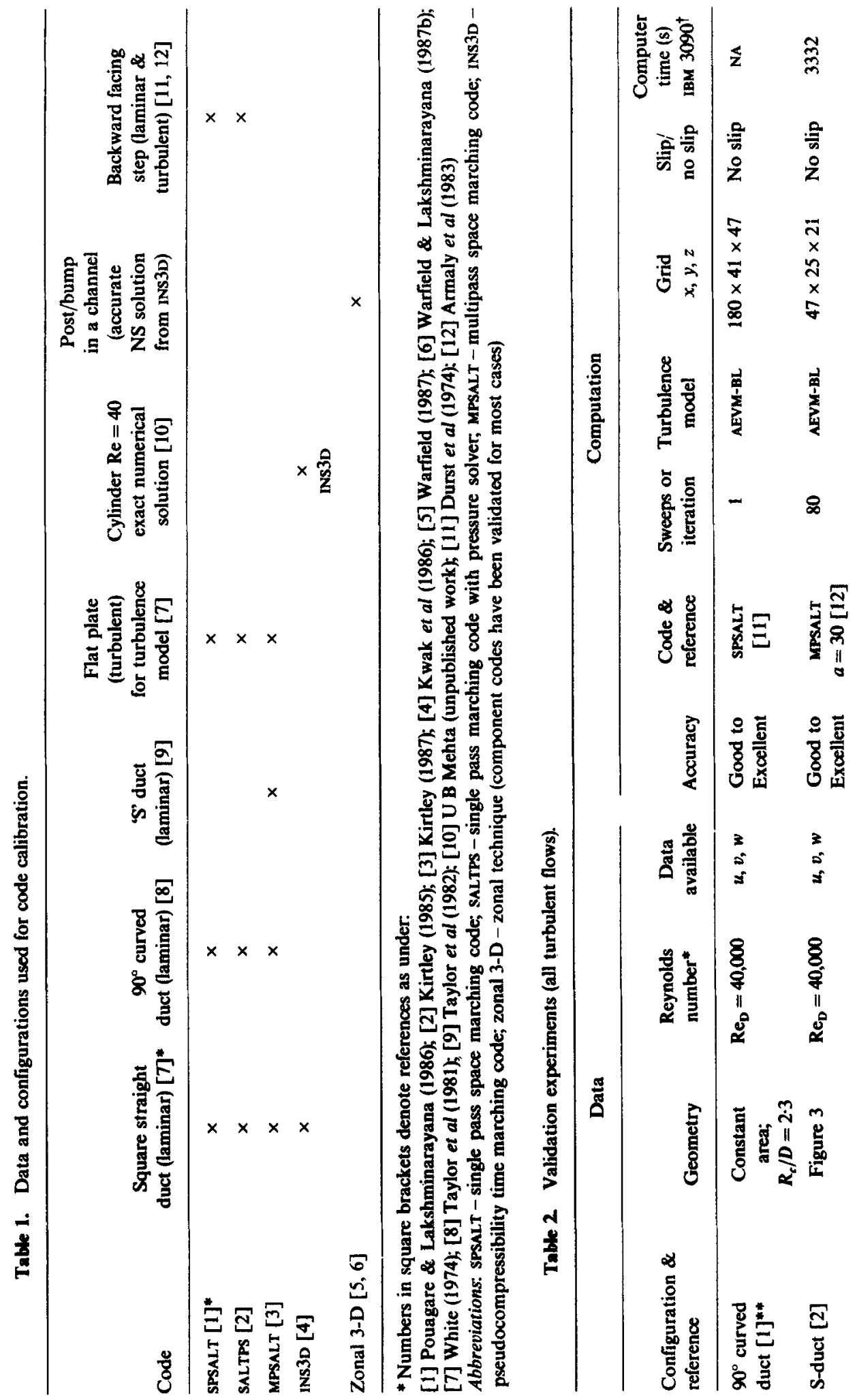




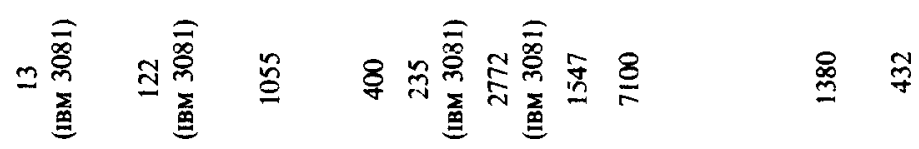

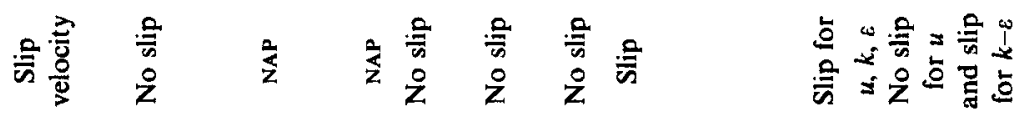

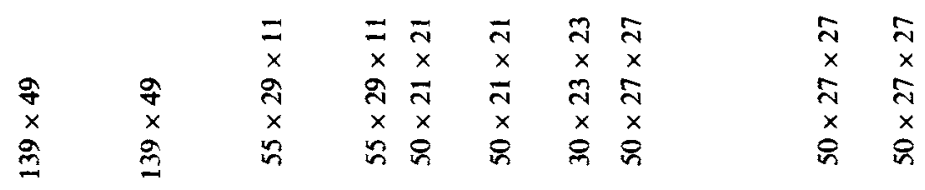

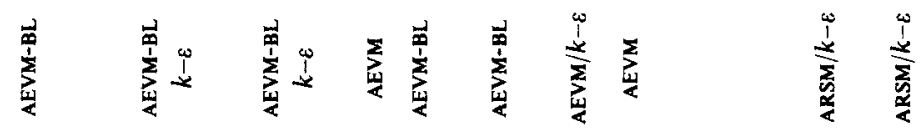

8 \&

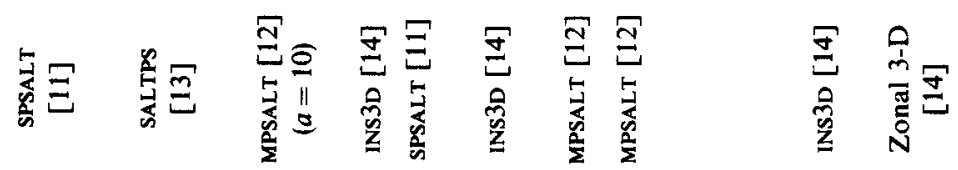

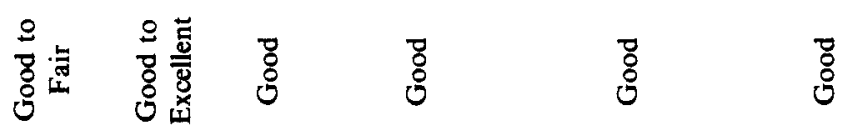

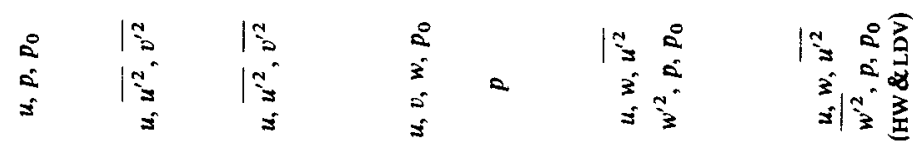

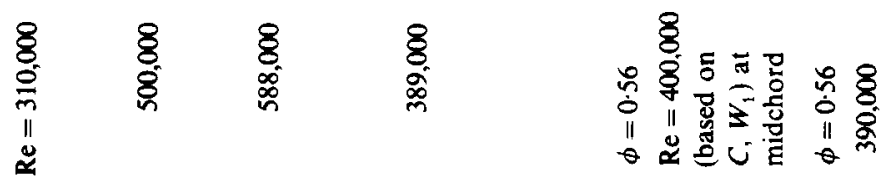

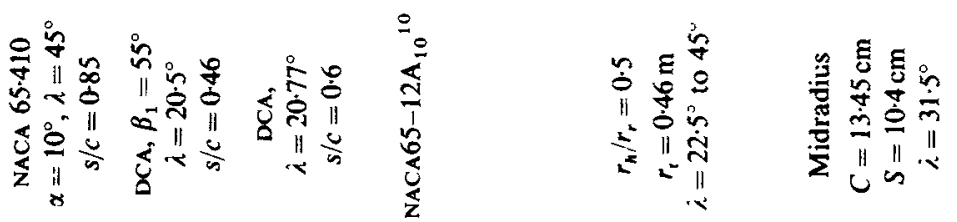

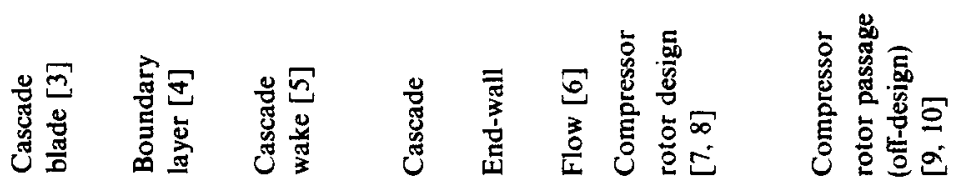

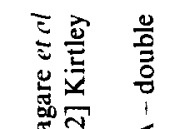

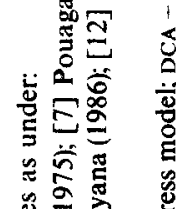

过焉

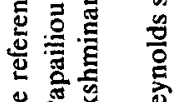

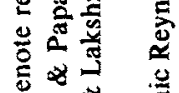

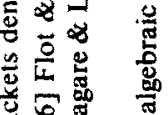

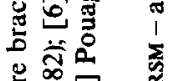

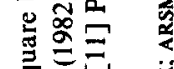

总吉芯

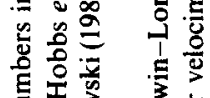

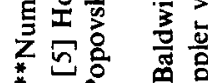

*

$\ddot{0} \approx$ क्षे

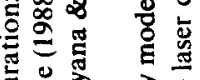

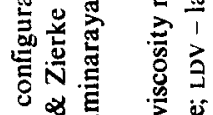

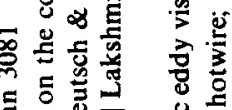

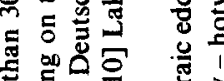

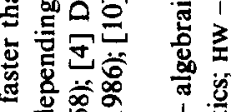

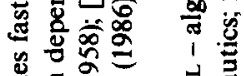

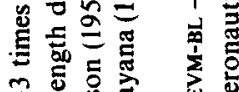

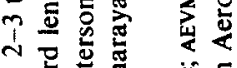

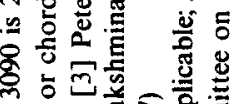

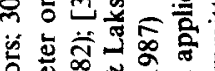

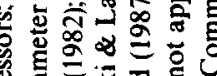

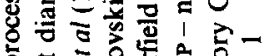

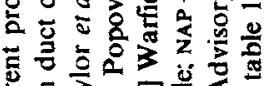

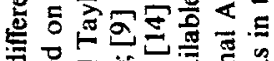

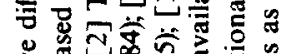

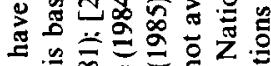

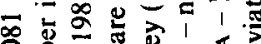

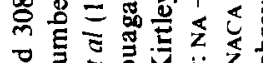

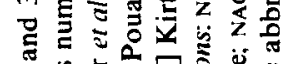

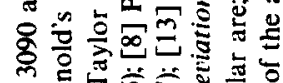

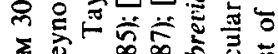

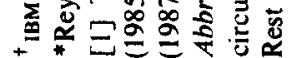




\section{Calibration of CFD codes}

The most important step in developing a good code is the calibration of the results against a known exact solution or very accurate data in simple configurations with laminar or turbulent flow. Simple configurations are essential to discover the errors associated with grid and boundary conditions, and laminar flow is essential to avoid problems with the turbulence model. It is also important to test complex turbulence models against well-documented classical turbulence data such as those for a flat plate boundary layer. A list of codes used in this paper, as well as the calibration configuration/data/analysis used, is shown in table 1.

\section{Validation of CFD codes}

Six different configurations and eight sets of data have been chosen for validating the computer code/techniques described in this paper. These are listed in table 2 and include both experimental and computational details. Several of these experiments were specifically designed to provide very accurate data for CFD validation. Among these are the data in a $90^{\circ}$ duct, S-duct, and the cascade blade boundary layer. It is prohibitively expensive to run all codes for all configurations, hence only selective runs are made to check the ability of these codes to predict complex flows, accuracy of turbulence models, and accuracy of near-wall flow prediction.

\section{$5.190^{\prime \prime}$ curved duct (3-D flow)}

The flow in a $90^{\circ}$ turning duct is highly three-dimensional, with substantial secondary flows due to the transverse pressure gradient. The algebraic eddy viscosity model was used in the SPSALT code to predict this flow (Taylor 1981). The assumed transverse pressure was approximated using a simplified radial equilibrium equation $(\partial p / \partial y=$ $u^{2} / R$ ), and it was assumed to be constant in the streamwise $(\xi)$ and spanwise $(\zeta)$ directions. The predictions are compared with the data at various locations in figure 2 . A better agreement can probably be achieved through the use of a finer mesh. Secondary velocity predicuions are good, especially near the surface. The overshoot in transverse velocity $(v)$ profile for $y / D=0.1$ is not predicted well; this is associated with the poor prediction of the streamwise velocity profile. It is well-known in secondary flow theory that the transverse velocities $(v)$ are caused by $\partial u / \partial z$ as well as by the flow turning angle. Hence there is a close correlation between $u$ and $v$ velocity profiles. Any inaccuracy in $u$ prediction will result in errors in $v$ predictions. Nevertheless, this computation has revealed that the constant area curved duct flows can be efficiently handled by a single pass marching technique, which requires over an order of magnitude less computation time than time marching or other iterative techniques.

\subsection{Flow in an S-shaped duct (3-D flow)}

The turbulent flow in an S-shaped duct was computed using the MPSALT code, as this case is characterized by strong transverse and streamwise pressure gradients. The results are compared with the data acquired by Taylor et al (1982); see table 2 for details. The geometry is shown in îgure 3 . No assumed pressure was used as an initial 

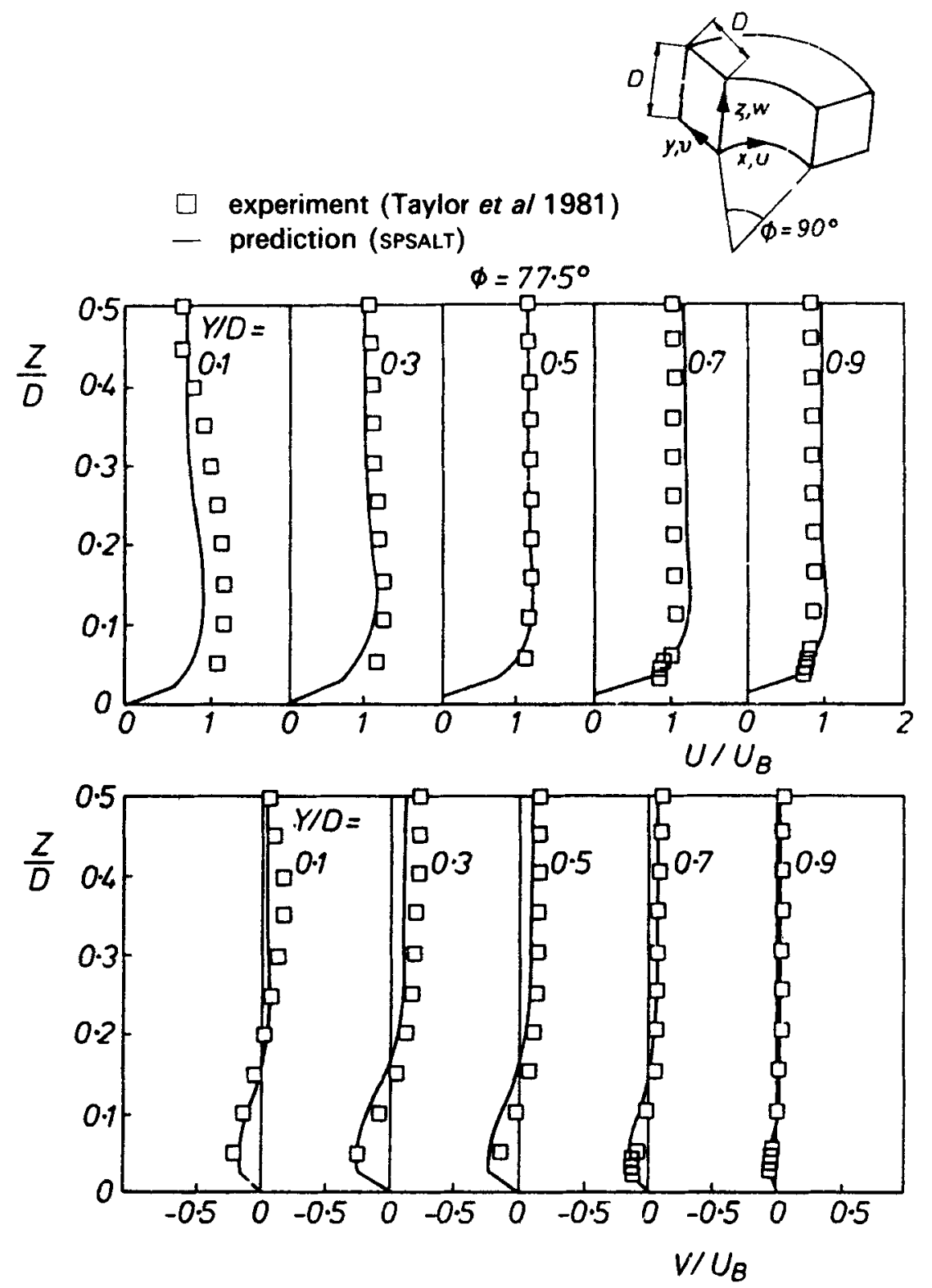

Figure 2. Velocity profiles at $\phi=77.5^{\circ}$ for a $90^{\circ}$ curved duct.

condition and the inlet flow was determined using the SPSALT code in the straight inlet section. The Reynolds number $(40,000)$ proved to be too low for successful application of the $k-\varepsilon$ turbulence model, thus the algebraic eddy viscosity model (AEVM-BL) was used. Nearly two orders of magnitude reduction in pressure residuals were achieved after 80 global iterations.

At the initial measurement location, the computed streamwise velocity profiles compared very well with the experimental data (Kirtley 1987). Thus the inlet conditions, so imoortant for space-marching methods, are accurately captured. At 


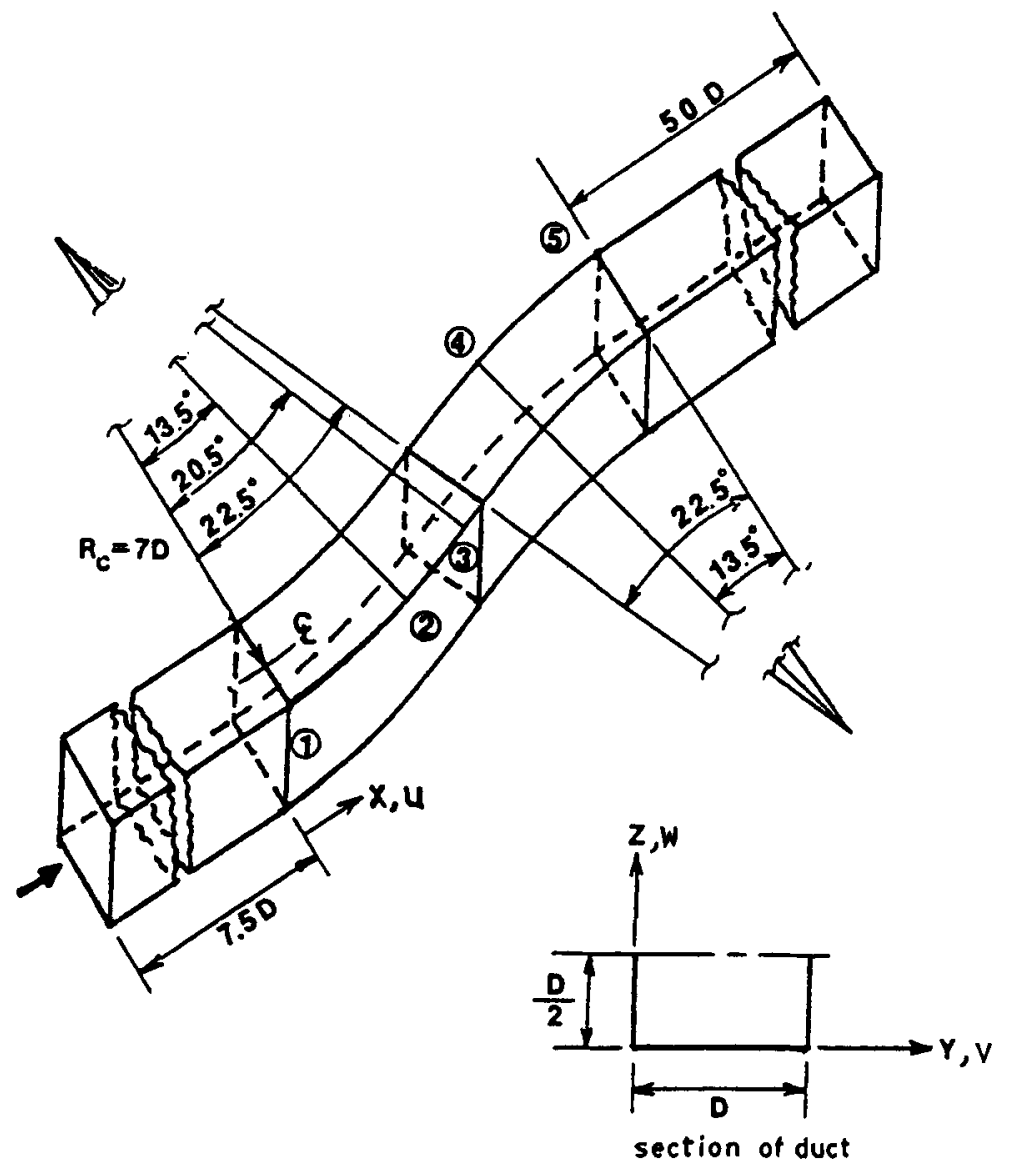

Figure 3. Geometry of the S-shaped duct.

station 2, the computed streamwise flow does not compare well with the experimental data near the centre of the duct (figure 4). Inspection of the profiles leads one to believe that there may be some computed mass surplus; however, the profiles very near the side walls were not measured and a conclusion can not be drawn. The computed mass flow did remain constant at convergence. The secondary flow at station 2 is predicted well (figure 4). The inviscid secondary flow is slightly overpredicted, yet the maximum flow turning in the end-wall viscous region is well-predicted. At station 4 (second bend of the duct), the computed streamwise and transverse velocity profiles compare well with the data (Kirtley 1987). The computed streamwise velocity profiles at station 5 show excellent agreement near the side walls and reasonable agreement near the centre of the duct (figure 5). The computed secondary flow profiles compare well with the experimental data except near the side walls, especially at $Y / D=0.9$. Here the measured transverse velocity has an S-shaped profile, and reversal of secondary flow has occurred (compare figures 4 and 5). Capturing the reversed secondary flow represents a very severe test case for any code. Due to lack of adequate data very near the wall, no comment can be made with regard to accuracy of prediction near the walls. The computed transverse pressure gradient is well-predicted everywhere, while the bulk pressure drop is not well-predicted (see figure 6). With excellent agreement 
data (Taylor et al 1982)

- predictions (MPSALT)
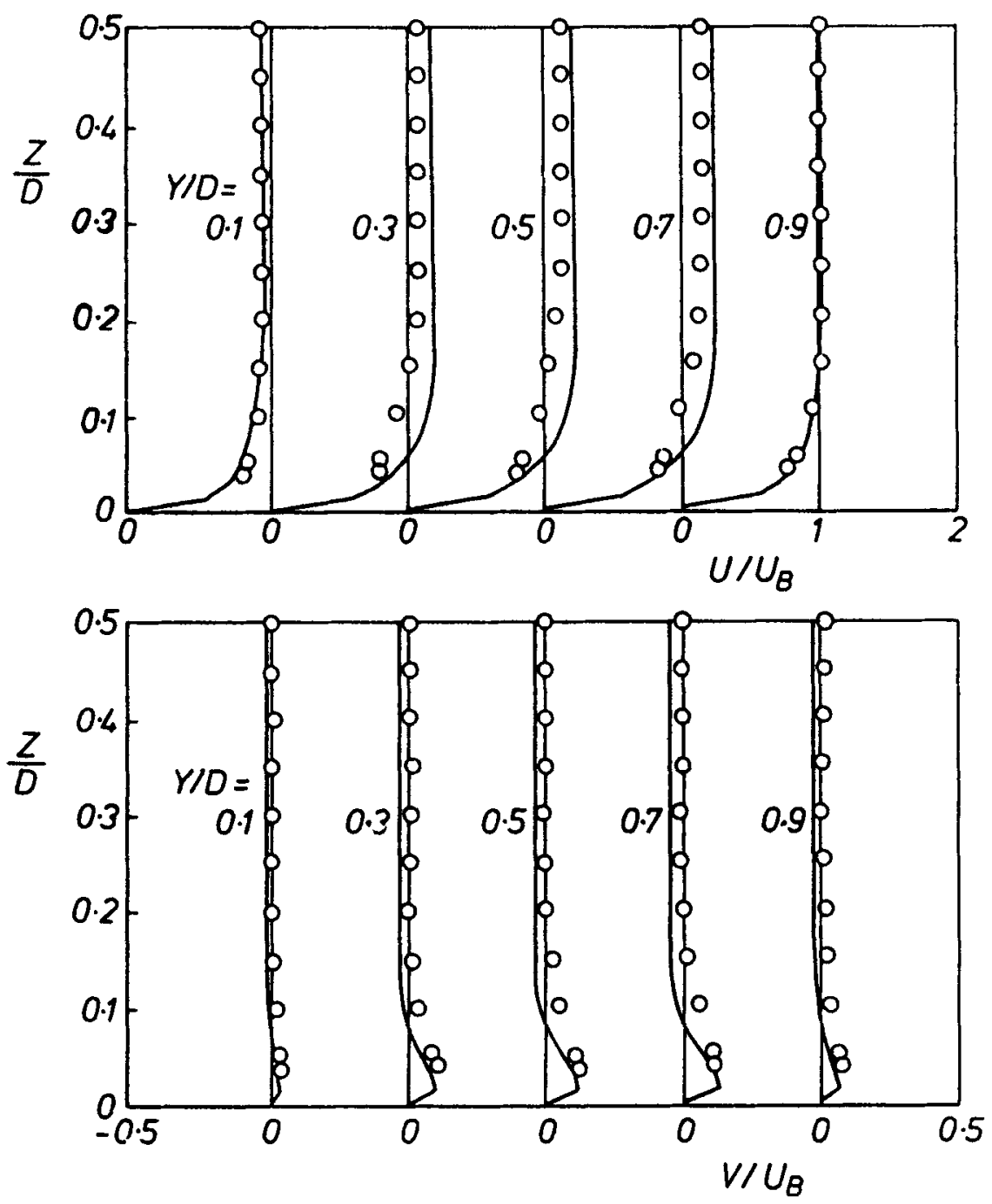

Figure 4. Velocity profiles for the S-duct at station 2 (see figure 3 for legend).

with the data for the laminar case for this same geometry (Kirtley 1987), the less-thanexcellent prediction of the turbulent flow implicates the turbulence model. The value of the near-wall velocity gradient is strongly dependent upon the turbulence model. Good predictions of the skin friction in two dimensions have been achieved by many users of the Baldwin and Lomax (1978) algebraic model. Therefore, simple extension of the two-dimensional model to three dimensions without regard for the actual physics of a three-dimensional boundary layer may be the reason for the reduced accuracy. 
data (Taylor et al 1982)

- predictions (MPSALT)
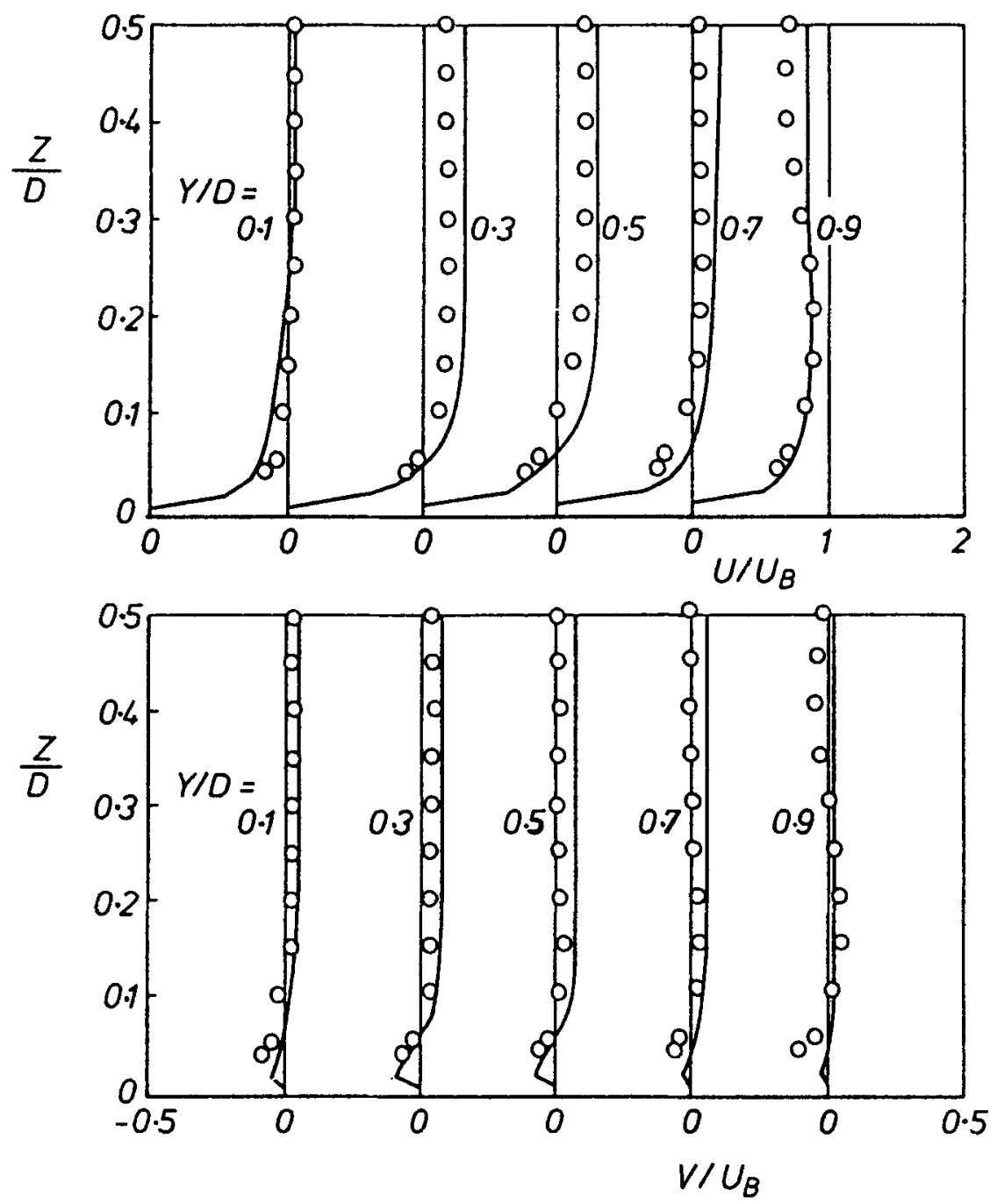

Figure 5. Velocity profiles for the S-duct at station 5 (see figure 3 for legend).

\subsection{Cascade blade boundary layer (2-D flow)}

In order to evaluate the ability of these codes to predict a cascade boundary layer which is two-dimensional, but is subjected to a large adverse pressure gradient on the suction side, the flow in Peterson's (1958) cascade was computed (table 2).

A slip velocity, as well as inviscid pressure from a panel code, is used in predicting the flow from a single pass space-marching code. The computed boundary layer profiles show excellent agreement with the data, except at $60 \%$ chord length (figure 7). 


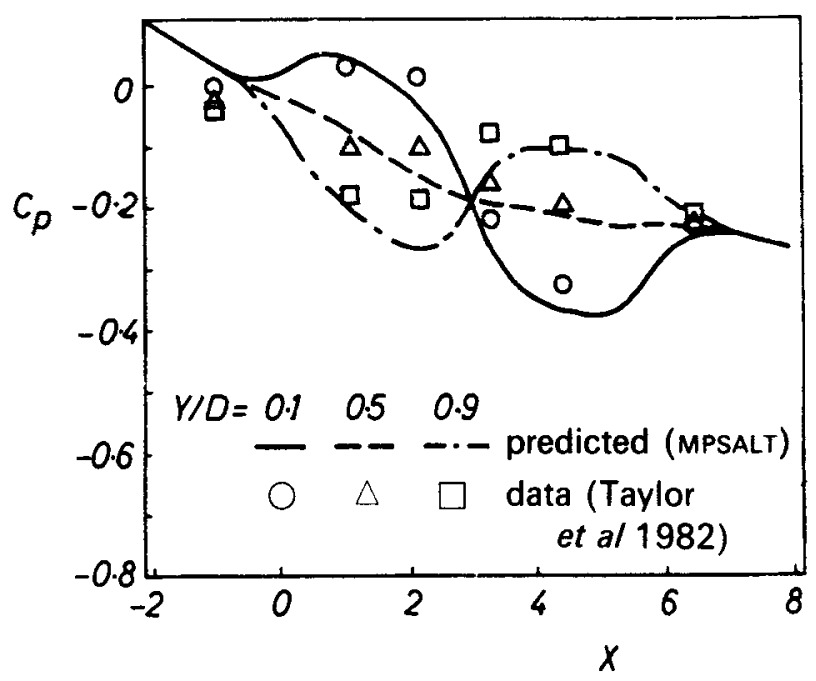

Figure 6. Coefficient of pressure for the S-duct (see figure 3 for legend).

It is thus evident that the major viscous effects, including the blade boundary layer, can be captured using an inviscid panel code and a single pass marching code. It is also clear that a simple algebraic eddy viscosity model is adequate for this flow.

\subsection{Separated cascade flow (2-D flow)}

Some benchmark data on separated cascade flow was acquired by Deutsch and Zierke (1988) for double circular arc blades (DCA) in a cascade (table 2). Mean and

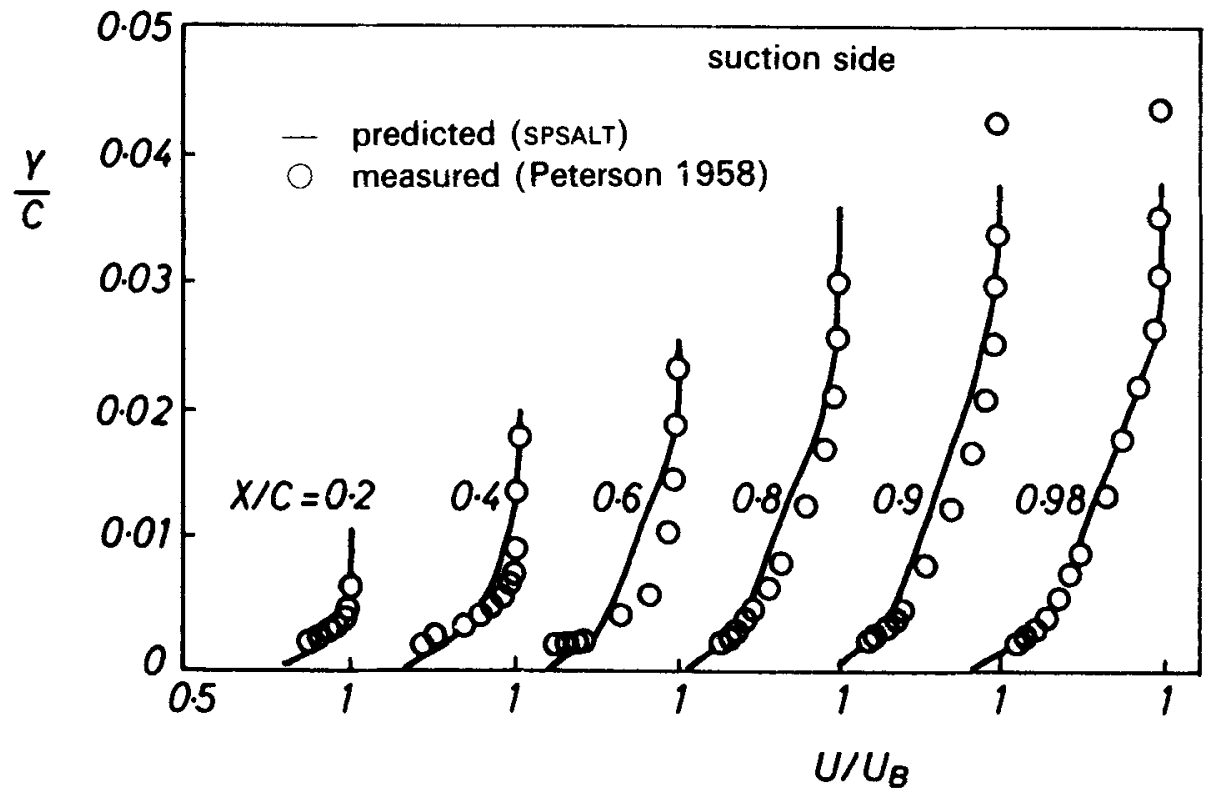

Figure 7. Velocity profiles on suction side of Peterson's cascade. 
fluctuating components of velocity were measured at various locations on the pressure and suction side, and in the wake, using a laser Doppler velocimeter. The flow was found to detach near the $80 \%$ chord location from the leading edge, and mean backflow velocities were measured even in the near-wake region. Therefore, this flow configuration is a good test case for SALTPS. The FLARE approximation was used in the separated zone.

Figure 8 shows the computed velocity profile for turbulent flow using the AEVM. For both the pressure and suction sides, the no-slip condition was used on the cascade blades. Up to the $53.6 \%$ chord location, the algebraic model does a good job of predicting the mean velocity profile. However, when the boundary layer detaches, computed at the $92.5 \%$ chord location, the mixing length hypothesis upon which the model was based breaks down and the prediction is poor. After four stations with
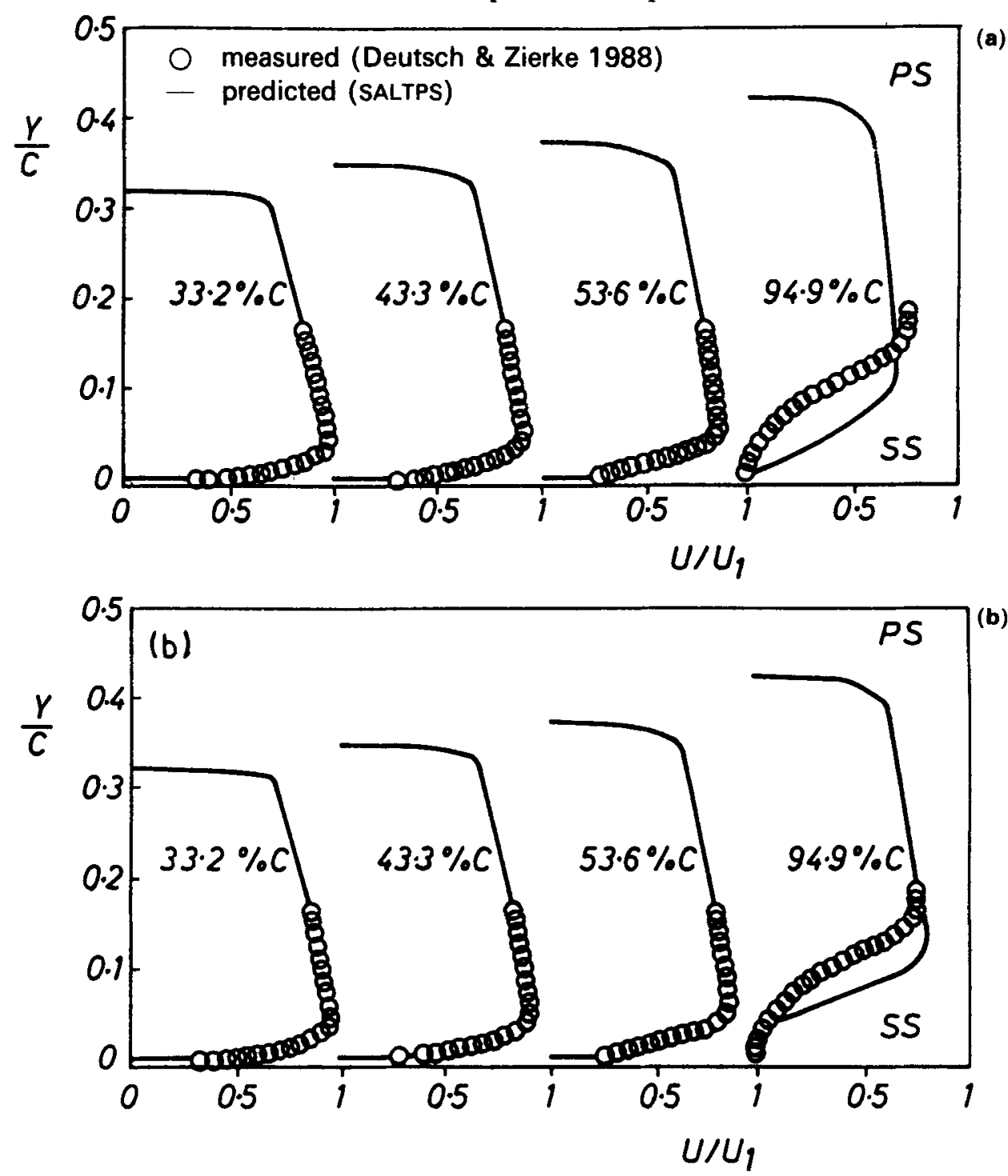

Figure 8. Velocity profiles for DCA cascade, $\operatorname{Re}=500,000$. (a) AEVM model, (b) $k-\varepsilon$ model. 
backflow velocities, the solution diverged, and so the computation was not carried out into the wake region. For all of the attached profiles, the inviscid core was wellpredicted, and no,problems were encountered either near the leading edge of the cascade blades or at the detachment point.

The two-equation $k-\varepsilon$ turbulence model was implemented in the hope that the inclusion of 'history effects' would improve the prediction in and around the backflow region. The velocity profiles computed using the $k-\varepsilon$ model are also given in figure 8 . In the backflow region, the $k-\varepsilon$ model gave much better results than the algebraic model but are still not good. The predicted detachment point was found to be $0.885 \mathrm{C}$ as compared to the actual point of $0.8 \mathrm{C}$. The extent of the backflow region is slightly over-predicted as is the magnitude of the backflow velocities.

The blade pressure distribution is shown in figure 9. The prediction on the pressure side is very good. However, on the suction side the prediction is rather poor near the trailing edge. One can see that the detachment point is computed much farther downstream than the actual point. The over-predicted backflow velocities have also contributed to a poor pressure prediction by causing a drop in pressure rather than a levelling off as measured. The upstream effect of the separation is substantial, and the ability of the SALTPS code to capture this effect is evident from the plot.

The above computation required only one sweep of the flow field. The Poisson equation for the pressure required 300 iterations to converge.

The technique is computationally efficient, and is a valuable tool for engineering analysis and design in industry. The results also indicate that neither the AEVM nor the

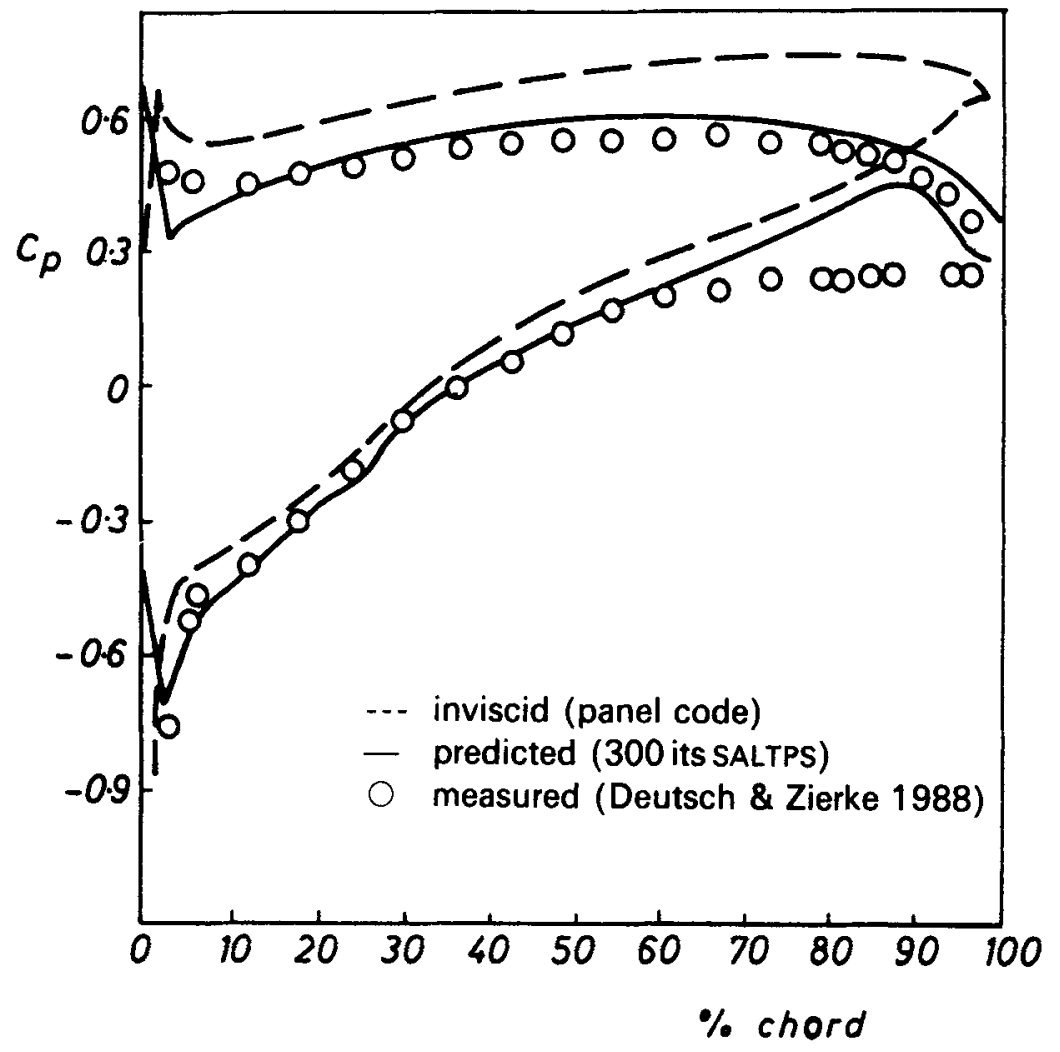

Figure 9. Pressure distribution for DCA cascade. 
$k-\varepsilon$ model is entirely suitable for predicting separated flows. However, the accuracy of the two models is almost identical for unseparated two-dimensional boundary layers subject to a pressure gradient.

\subsection{Compressor cascade wake flow (2-D)}

This represents a free shear layer and is a good test case for validation of turbulence models. The MPSALT and INS3D codes were used to compute wake flows measured by Hobbs et al (1982) using a hot film probe for the near wake and a five-hole probe for the far wake (table 2). The inviscid pressure computed from the panel method was used as the initial pressure field to compute both the boundary layer and the wake development using the MPSALT code. A no-slip condition was used on the blade surfaces, and the slip condition was applied on the end-wall in an effort to achieve two-dimensionality.

The flow was computed with both the algebraic eddy viscosity model and the $k-\varepsilon$ turbulence model. Comparison of the computed boundary layer profiles $\left(x / C_{x}=\right.$ $-0.029)$ at the trailing edge and the wake profiles with the experimental data is given in figure 10. The boundary layer at the trailing edge is in good agreement with the data. The computed velocity profiles do not compare well with the experimental data near the wake centreline. The far wake is only adequately predicted. The wake centreline position is below the measured position and the wake-spreading is less than measured. The wake-spreading is a strong function of the turbulence and the ad hoc approximations made in the algebraic turbulence model may contribute to the poor prediction.

The wake velocity profiles computed with the $k-\varepsilon$ model in the MPSALT code are also shown in figure 10. Again the boundary layer profiles are reasonably wellpredicted; however, the near-wake profiles show the same lack of agreement with the centreline data as the computation with the algebraic model. In the far wake, the advantages of the $k-\varepsilon$ model are clear, with excellent agreement between computation and measurements. The wake centreline position, as well as the wake-spreading, is accurately predicted even though the near-wake predictions were not as accurate. This leads one to believe that the far-wake solution is not strongly influenced by the near wake. Overall, the prediction using the $k-\varepsilon$ model is superior to the prediction using the algebraic model for all regions of the flow.

The predictions from the Ins3D code, using the AEVM-model, is shown compared with the corresponding predictions (with the same turbulence model) and data in figure 10 . The accuracy of the boundary layer prediction is equally good in both, but the inviscid velocities on the suction side are captured more accurately with the timemarching code. The prediction of the wake is comparable to that from the MPSALT code and suffers from the same deficiencies mentioned earlier. The wake centreline velocity and wake trajectory are not properly captured from either code with the AEVM turbulence model.

\subsection{Cascade end-wall flow (3-D flow)}

The techniques described earlier (SPSALT, INS3D, MPSALT) were used to compute the three-dimensional turbulent flow in the end-wall of a cascade (Flot and Papailiou 1975) (table 2). The inviscid pressure from a panel code is used as the assumed pressure 

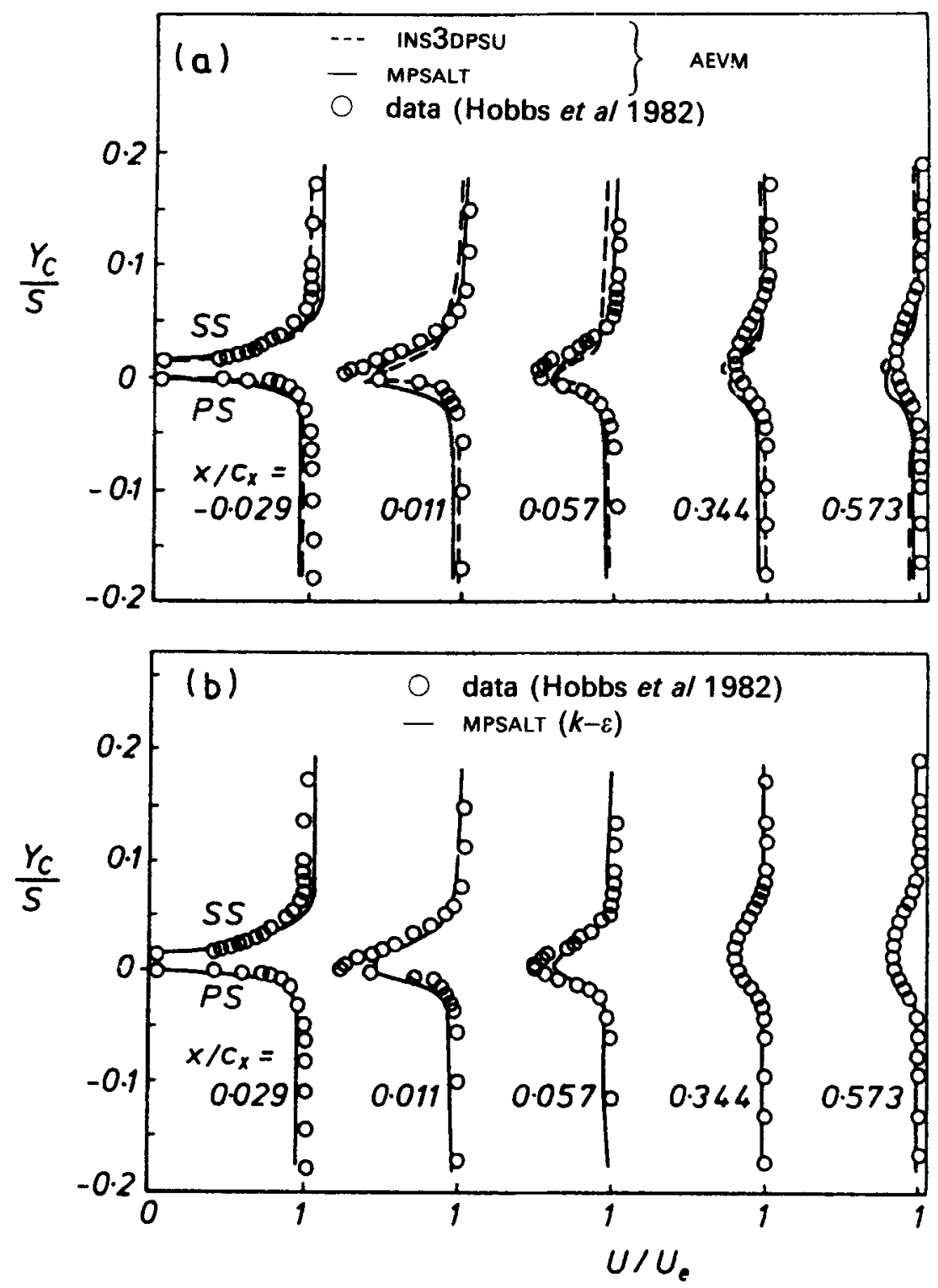

Figure 10. Wake profiles for Hobbs' cascade.

in the SPSALT code, while no such assumed pressure is required for MPSALT and INS3D codes.

The space-marching method was effective in predicting the streamwise velocity profiles at each station, but the prediction is particularly good near the suction side stations (figure 11). On the other hand, the profile computed by the time-marching method represents a very thin blade boundary layer $y / s=0.11$ which contributes to a poor prediction there. Unfortunately, there is no experimental data near the blade/end-wall corners to evaluate predictions in this region. The time-marching method is more successful in predicting the end-wall velocity profiles near the pressure side. Here the space-marching method is somewhat deficient. 

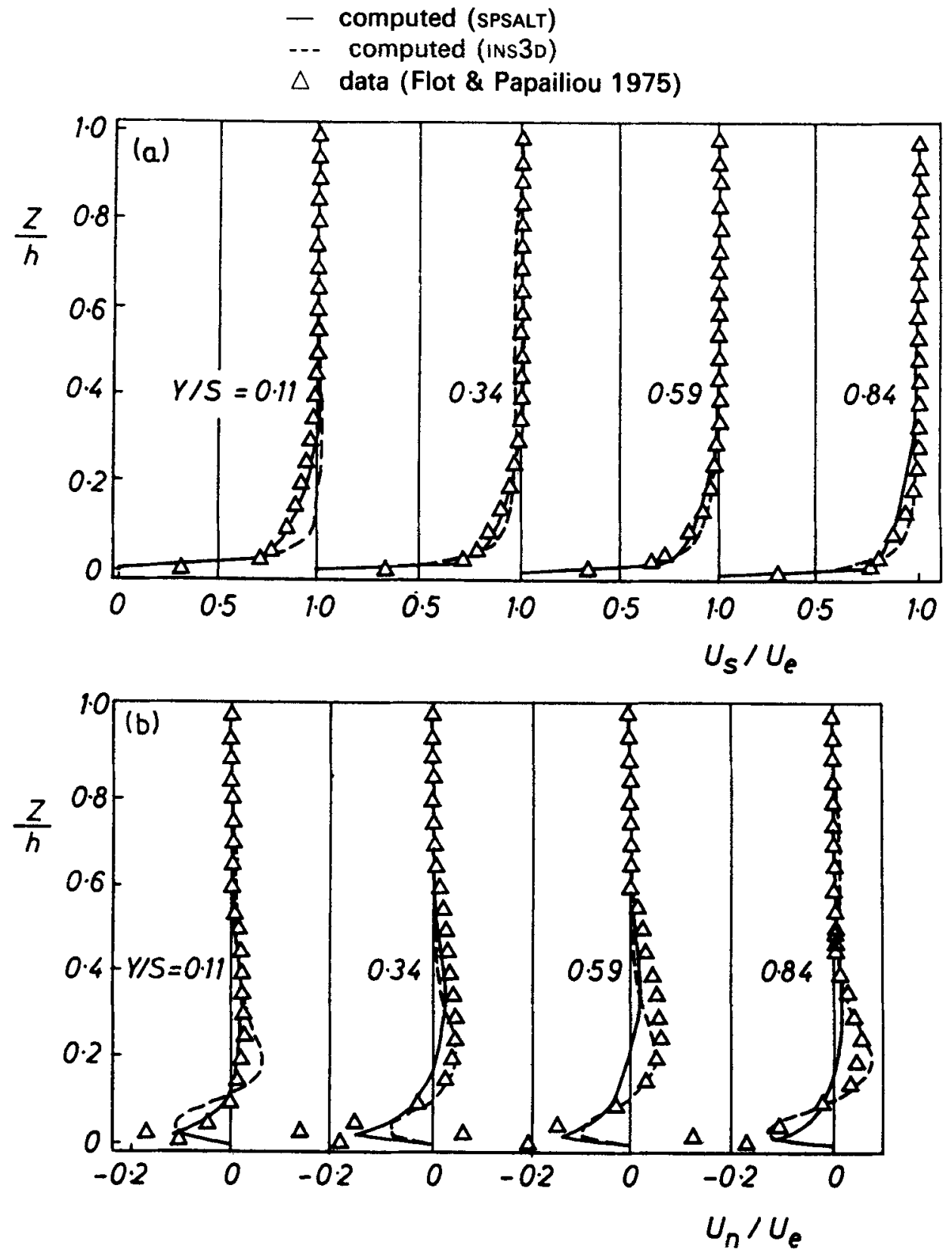

Figure 11. End-wall velocity profiles at $44 \%$ chord ( $y$ is the tangential distance from the suction surface).

A plot of the secondary velocity profile indicates that the time-marching method (INS3D) predicts the underturning of the flow in the outer reaches of the end-wall boundary layer better than the space-marching method (figure 11). The spacemarching method performs better very near the end-wall where the overturning of the flow is qualitatively better predicted. However, the magnitude of the peak of the overturning is not captured, especially farther downstream. Again the predictions 
show that the space-marching method performed better near the suction side, while the time-marching method performed better near the pressure side.

The time-marching solution required approximately 12 times as much computational effort as the space-marching technique. The space-marching code requires $1.5 \mathrm{Mb}$ of storage while the time-marching code requires $3.6 \mathrm{Mb}$.

With respect to prediction accuracy, both techniques had their strong points, and showed better predictions for different parts of the flow field. Overall, the spacemarching method appeared to have an edge in predicting this viscous flow. However, it must be noted that the grid used was more suitable for the space-marching method than for the time-marching method. Such a grid could not be used for a blunt leading edge airfoil, which precludes the use of the space-marching method. For the spacemarching method, as long as the flow field at the preceding streamwise station is regular, the eddy viscosity field remains smooth and regular and leads to a successful computation. During the iteration process of the time-marching method, the flow field may not be regular, which can lead to a spurious eddy viscosity field. This can be destabilizing and must be handled very carefully when algebraic models are used. This problem appears to be most acute in flow regions containing interacting boundary layers.

The subject of smoothing is important when comparing these two techniques. The space-marching method includes no smoothing terms, while the time-marching method utilizes both explicit and implicit smoothing in the computation of the flow. The concurrent effects of smoothing and pseudocompressibility in computing a large Reynolds number turbulent flow with the considered time-marching method are not easily defined; more applications of the technique to such cases will further elucidate this matter.

The MPSALT code has also been validated against this data, and agreement with the data is found to be excellent (see table 2 for computational efficiency) even though the computational time is the same as time-marching, but an order of magnitude more than the SPSALT.

These results provide an interesting dilemma. The Navier-Stokes solution is not necessarily the most accurate one, as error due to numerical technique, artificial dissipation and smoothing may compensate for the increased accuracy of the equations. The space-marching method provides good predictions. One word of caution. In turbine cascades, where large flow separation (end-wall) and horseshoe vortices are encountered, the SPSALT technique failed to capture the elliptic effects associated with these features. For incompressible flow through cascades, without any end-wall separation, the SPSALT code provides excellent predictions.

\subsection{Flow in a compressor rotor passage}

One of the most complex internal flows is the flow in turbomachinery rotor passages. It is three-dimensional, turbulent and is subject to the influence of rotation and curvature. This is a very severe test case for 3-D viscous codes. Comprehensive data on pressure, velocity field, including the blade boundary layers from hub to tip, leading to the trailing edge is available for the Penn State low-speed compressor (table 2). The rotor blade boundary layer was acquired with a miniature rotating ' $x$ ' configuration probe, and the inviscid and end-wall flow data was acquired with either a laser doppler velocimeter or a rotating three-sensor hot wire probe. Two sets of data are available. 
(1) The blade boundary layer, end-wall flow field and inviscid flow field in the entire flow passage and the wake is reported in Pouagare (1984) and Pouagare et al (1985) for design conditions. This flow was computed using the MPSALT code. Details of computation and experimental data are listed in table 2.

(2) Details of data on the blade boundary layers and inviscid flow field, end-wall flow in the entire rotor passage at the peak pressure rise coefficient $(\phi=0.50)$, where the boundary layer growth is substantial, can be found in Popovski \& Lakshminarayana (1986) and Lakshminarayana \& Popovski (1987). This flow was computed using INS3D and zonal 3-D codes.

In both cases, the flow is incompressible. For the design case $(\phi=0.56)$, the MPSALT code used inviscid inlet conditions at the leading edge, and the initial inviscid pressure was generated by stacking multiple two-dimensional solutions from the panel method. Turbulent slip conditions were used on all the blade surfaces. Detailed comparisons are given in Kirtley \& Lakshminarayana (1988) and will not be repeated here. The suction surface boundary layers are predicted well, except near the tip regions. It is important to remember that the tip clearance effect has not been included in the computation and the measured data at this location may indeed include the interaction of the leakage jet with the blade boundary layer. The predictions are not as good on the pressure side, as the boundary layers are very thin (1-4 mm) and both measurements and computation are subject to error.

At the peak pressure rise coefficient $(\phi=0.50)$, the blade boundary layers are approximately twice as thick as those at $\phi=0.56$. Hence the data is more accurate and comprehensive. This case was computed using both the INS3DPSU code and the zonal 3-D code. Both techniques used the same grid, the same turbulence model and the same computer so that proper comparisons can be made.

The zonal method for this application consisted of an elliptic zone extending from the leading edge to the $25 \%$ chord location. The parabolic zone continued from the $25 \%$ chord location to the trailing edge. Both zones extended from blade to blade and from hub to tip. The wake was not computed for this flow coefficient. Only one sweep of the zonal method was utilized; the combination of zones with the elliptic zone upstream and the parabolic zone downstream reduces the need for multiple zonal sweeps due to the inability of the parabolic solution to influence the upstream elliptic solution.

A critical difference between the zonal and the benchmark predictions exists for this case. The benchmark time-marching solution (INS3DPSU) utilized turbulent slip velocity conditions on the blade surfaces, whereas the space-marching zone solution (SPSALT) utilized the no-slip condition. Otherwise, boundary conditions are unchanged. The prediction of the streamwise velocity on the suction side of the rotor blade is shown in figure 12. In this case, the INS3D solution shows quite good agreement with the data, especially near the trailing edge of the blade, while the zonal solution shows better agreement near the leading edge. The zonal solution consistently predicts a thinner boundary layer than the benchmark solution for both suction and pressure surfaces. In this case, the boundary layer edge velocity predicted by the zonal solution matches the data.

Radial velocity predictions on the suction side are shown in figure 13 . The correct qualitative trends are predicted in both solutions, but the zonal solution is clearly superior in most chordwise and radial locations. The no-slip treatment and the lack of smoothing in the parabolic zone of the zonal solution increases the quality of the 
$\diamond \triangle \square$ data (Lakshminarayana \& Popovski 1987)

-... zonal 3-D

- INS3DPSU

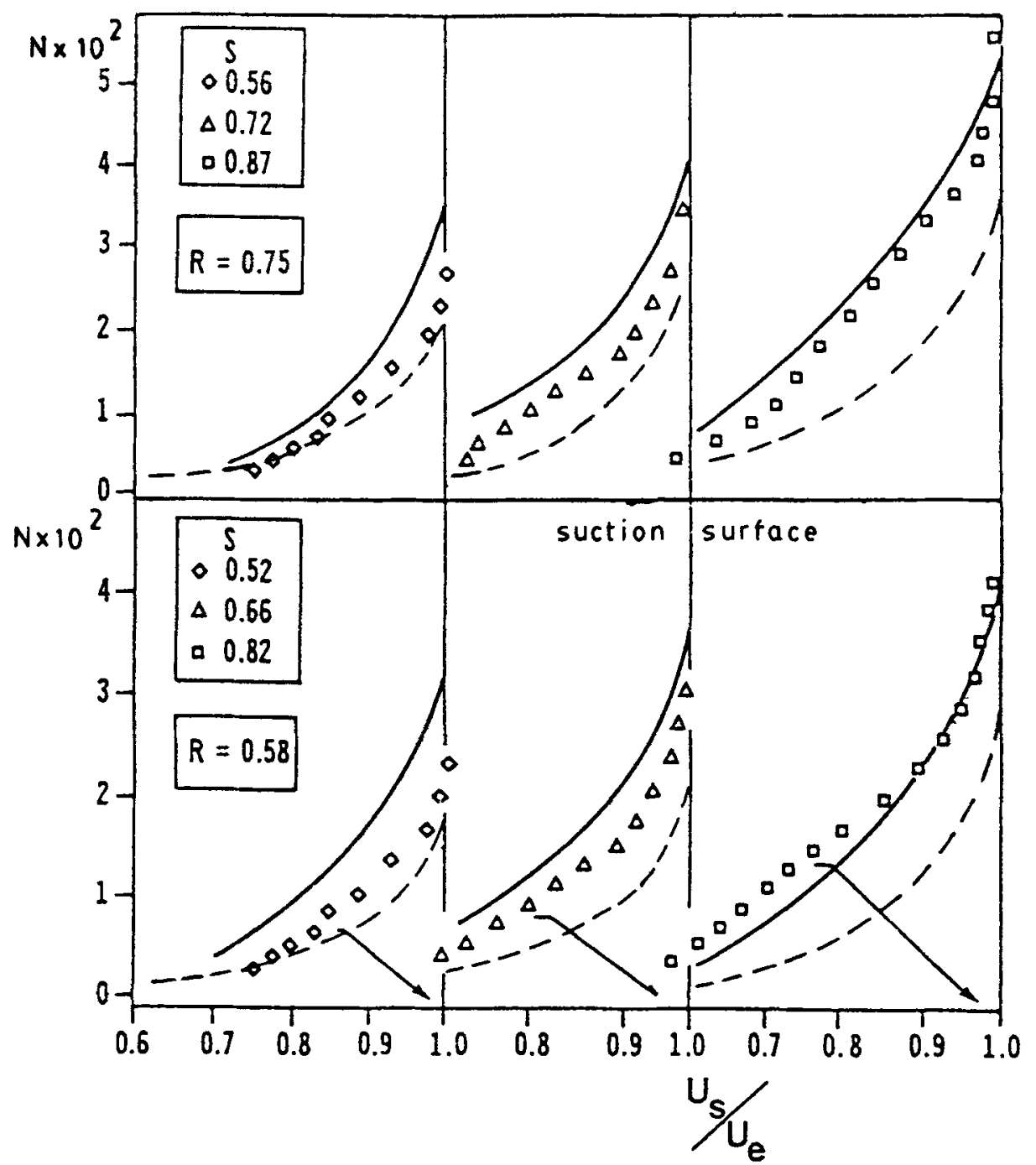

Figure 12. Streamwise velocity profiles on suction surface of compressor rotor blade at $R=0.58$ and $R=0.75$.

zonal prediction of radial flows. The benchmark solution gives inadequate definition of the radial flow profiles, missing all steep gradient regions and consistently underpredicting the maximum radial velocities near the wall. The mid-span radial flow prediction in the zonal case is particularly good, with both peak values and streamwise trends accurately predicted.

It should be remarked here that we do not imply that the Ins3DPSU code, which was used as a benchmark solution, is less accurate than the zonal code. If no-slip conditions are used in the INS3DPSU code, it should provide as accurate results as did the zonal 3-D code. But the smoothing in the INS3DPSU code should affect the solution 


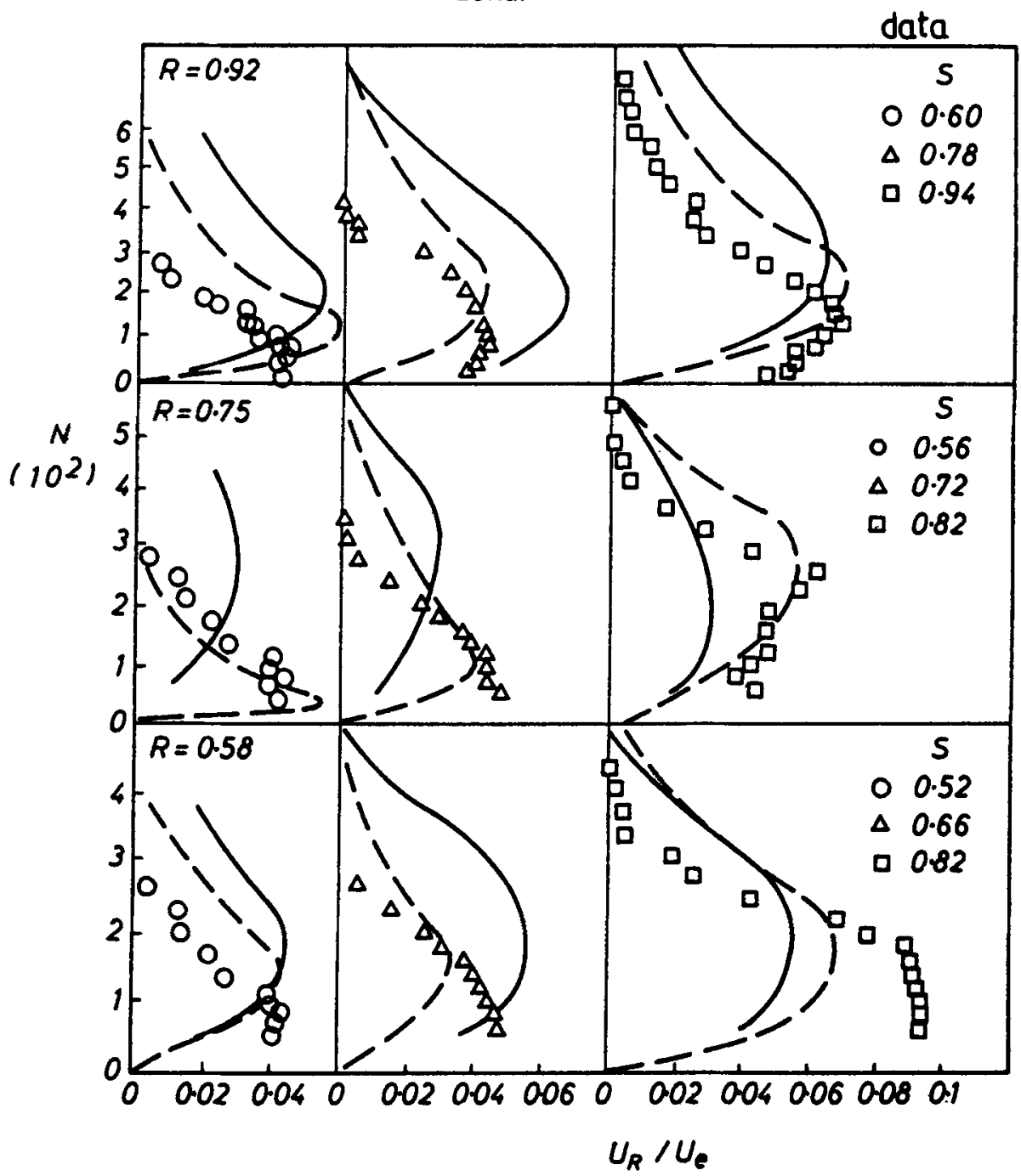

Figure 13. Radial velocity profiles on suction surface of compressor rotor blade.

near the wall to a greater extent with no-slip boundaries, giving poor boundary layer predictions.

The composite velocity predictions are shown in figure 14 for $R=0.75$. The overall features of the flow field have been predicted by both solutions, with the best predictions occurring near the trailing edge. The overshoot of the boundary layer edge velocity on the pressure side for the zonal solution is one distinct feature which is readily apparent in the composite velocity plots. The prediction of the composite velocity is a very important result of a Navier-Stokes solution as it verifies the coupling effects of the viscous and inviscid flow features in establishing the overall pressure and velocity field. Both solutions have shown this capability.

The success of the zonal method (69\% reduction in computer time) for this case, in 


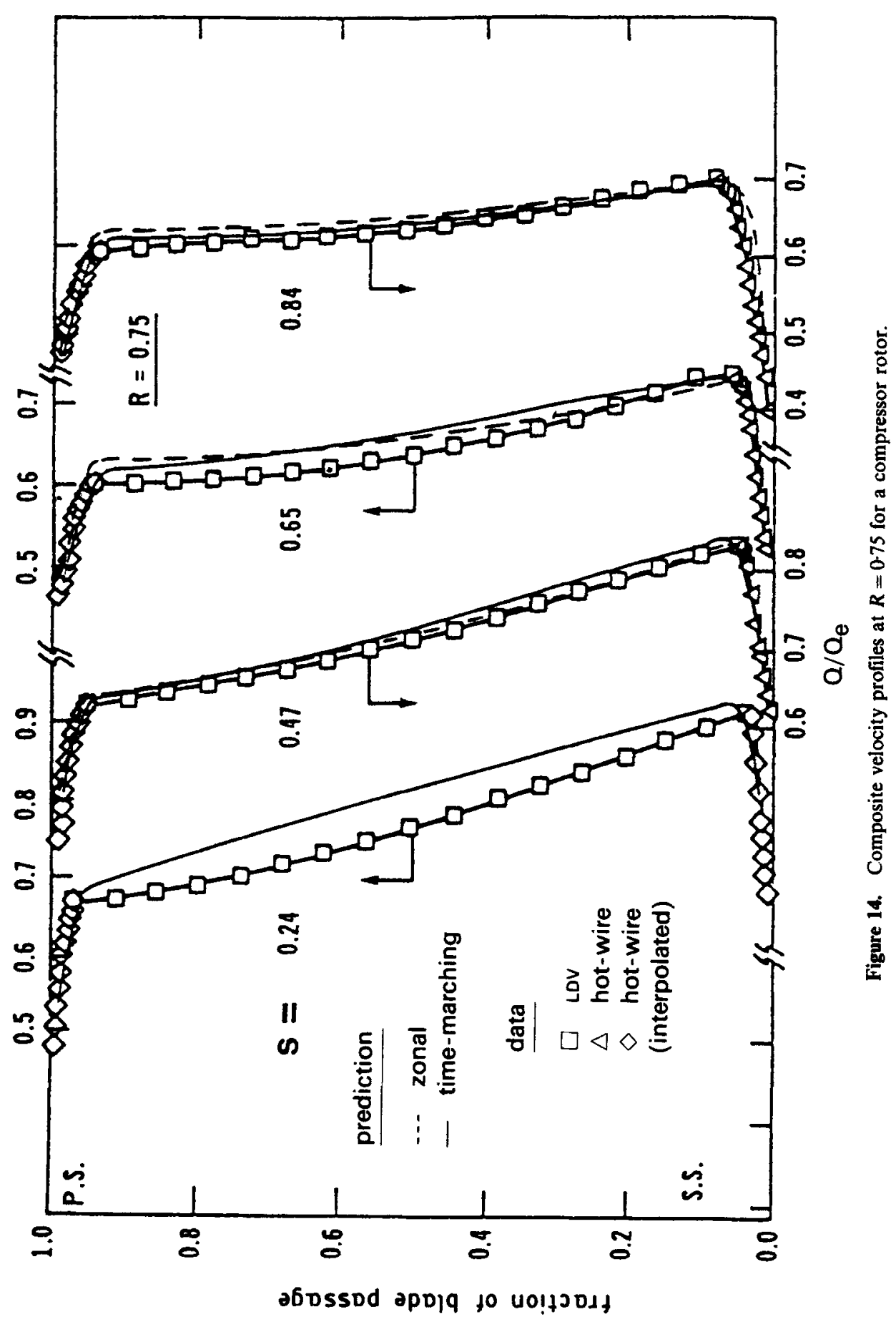


both efficiency gain and in solution quality, makes it promising as a method for application to other complex turbulent flows where space-marching methods cannot be applied over the entire flow.

\section{Concluding remarks}

We have demonstrated, through systematic calibration and validation, that simple and efficient techniques can be developed to predict internal flows. It is not necessary to use the full Navier-Stokes code for all situations. For incompressible flows, the classical methods available (e.g., the panel method for lifting body, inviscid analysis for turbomachinery flows) can be coupled with the space-marching methods to resolve the viscous layers. In situations where both transverse and streamwise pressure gradients are present, it is necessary to employ more accurate methods of capturing the pressure field.

The MPSALT technique, which utilizes the pseudocompressibility concept, is found to be accurate and unconditionally stable for a multipass global iteration procedure. Further refinement will require finer grid, modelling, and acceleration schemes such as multigrid techniques.

The zonal equation method, which is an efficient method for resolving viscous flows in rotors, single-stage and multi-stage turbomachinery, has been developed, calibrated and validated. This technique combines the efficiency of the space-marching technique and the accuracy of time iterative methods. The $69 \%$ savings in time for the zonal compressor rotor flow solution was particularly impressive, as the solution compared better with the data than did the time-marching benchmark solution. The application of these techniques to multi-stage turbomachinery is promising. But the zonal technique needs improvement in the area of the adaptive buffer zones, and it could possibly include analytical solutions in some regions. The efficiency of this code is coupled to those of the component codes. The interaction of the zonal equation method with possible zonal turbulence modelling application and solution adaptive and/or zonal grid generation would provide a long-term goal of developing efficient techniques for all internal flows, including multi-stage turbomachinery, combustion flows etc.

It has been demonstrated that the algebraic eddy viscosity model is adequate for two-dimensional flows with moderate streamwise pressure gradients. The model was not successful for 2-D wake flows. The spreading of the wake centreline trajectory and the wake centreline velocity are strong functions of the turbulence. Hence, it is not surprising that the wake flows need more physically realistic models. The area which needs the most attention is the development of more accurate transition and turbulence models as well as the application of existing transition and turbulence models to the unique probiems of turbomachinery.

The area that needs immediate attention is benchmark-quality data in complex internal flows such as turbine end-wall flows and rotor flows. These measurements are not only complex, but very expensive. It is doubtful whether the same accuracy as benchmark-quality data (ducts, airfoils, bends) can be achieved in a rotor. New measurement techniques for resolving the wall layers, end-wall flows and corner flows are essential before one can confidently establish the accuracy of these codes. A comprehensive data set should include not only three components of mean velocity, 
temperature, but also turbulence qualities such as the $\overline{u_{i}^{\prime} u_{j}^{\prime}}, \overline{u_{i}^{\prime} T^{\prime}}$ spectrum, wall shear stress and heat transfer. Similarly, accurate data on transition and separation on a three-dimensional configuration are lacking.

The research work on computation was sponsored by the David Taylor Naval Ship Research and Development Center with Dr D Fuhs as the technical monitor, and NASA Lewis Research Center with Dr P Sockol as the technical monitor. Numerous discussions with Drs P Sockol and D Fuhs are gratefully acknowledged. Drs T R Govindan, M Pouagare, and J Gorski were responsible for the development of SPSALT and the earlier codes, and their assistance is gratefully acknowledged.

\section{List of symbols}

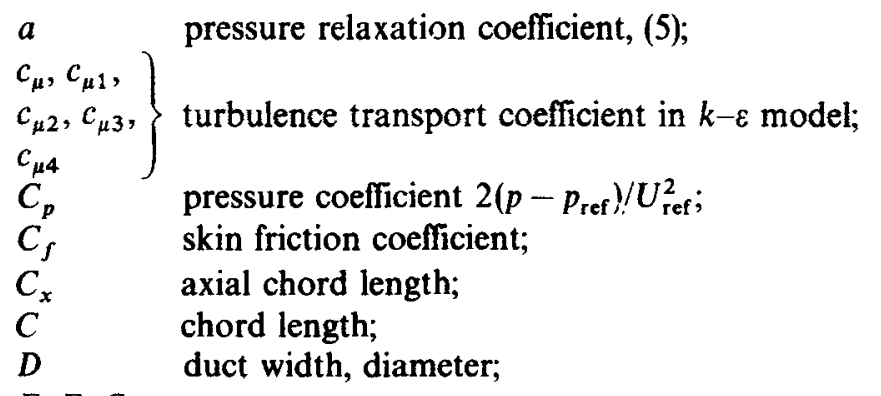

$E, F, G, q$,

$S, B, S_{m} \quad$ vectors in (1);

$2 h \quad$ blade height;

$k \quad$ turbulence kinetic energy;

p static pressure;

$P \quad$ production of turbulence energy;

$Q \quad$ total velocity (relative velocity for a rotor);

$r \quad$ radius;

$R, R^{\prime} \quad$ residuals;

$\operatorname{Re} \quad$ Reynolds number;

$R_{c} \quad$ radius of curvature of duct;

$S, N, R$ streamwise, normal and binormal directions, normalized by blade chord for $S, N$, normalized by tip radius for $R$;

$S \quad$ cascade blade space/streamwise distance from leading edge;

$T, T^{\prime} \quad$ mean and fluctuating temperature;

$u, v, w \quad$ velocity in $x, y, z$ directions (for curved duct see figure 2 , for S-duct see figure 3; for the definition of velocity components, $u$ is the streamwise and $v$ is the cross-flow velocity);

$u_{B} \quad$ bulk velocity;

$U_{s}, U_{n}$, relative velocity in $S, N, R$ system for cascade end-wall and rotor flows

$U_{R} \quad\left(U_{s}\right.$ is the streamwise velocity, $U_{n}$ is the velocity in blade-to-blade direction, $U_{R}$ is the radial velocity);

$u^{*} \quad$ friction velocity $\left(\tau_{\text {wall }} / \rho\right)^{1 / 2}$; 


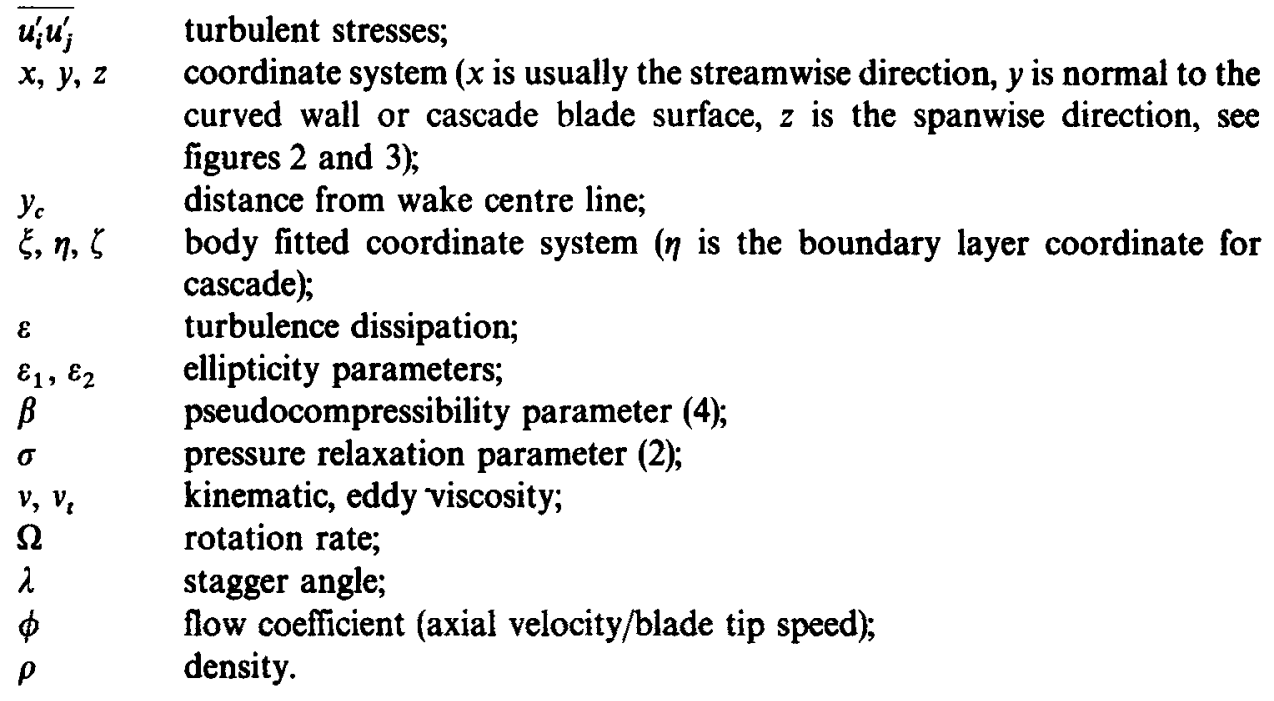

\section{Subscripts}

$\begin{array}{ll}1 & \text { inlet, first grid point; } \\ t, h & \text { tip, hub; } \\ \text { ref } & \text { reference; inlet velocity; } \\ e & \text { edge velocity; } \\ \text { ss, ps } & \text { suction, pressure surface. }\end{array}$

\section{References}

AGARD 1985 3D computational techniques applied to internal flows in propulsion systems, AGARD LS 140 Armaly B F, Durst F, Pereira J C F, Schonung B 1983 J. Fluid Mech. 127: 473-496

Baldwin B S, Lomax H 1978 Thin layer approximation and algebraic model for separated turbulent flows, AIAA 78-257

Briley W R, McDonald H 1980 J. Comput. Phys. 34: 54-73

Chorin A J 1967 J. Comput. Phys. 2: 12-26

Deutsch S, Zierke W C 1988 J. Turbomachinery 109: 138-152

Durst F, Melling A, Whitelaw J H 1974 J. Fluid Mech. 64: 111-128

Flot R, Papailiou K 1975 Couches Limites et Effets D'Estremities Dans Les Turbomachines, METRAFLU, contract DR. M.E. 73/373

Galmes J M, Lakshminarayana B 1984 AIAA J. 22: 1420-1428

Gorski J J, Govindan T R, Lakshminarayana B 1985 AIAA J. 23: 685-692

Govindan T R, Lakshminarayana B 1988 Comput. Fluids 16: 21-39

Hobbs D E, Wagner J H, Dannenhoffer J F, Dring R P 1982 Experimental investigation of compressor cascade wakes, ASME Paper 82-GT-299

Jones W P, Launder B E 1973 J. Heat Mass Transfer 16: 1119-1130

Kirtley K R 1985 Computation of internal incompressible separated flow using a space-marching technique, MS thesis, Department of Aerospace Engineering, The Pennsylvania State University

Kirtley K R 1987 A coupled parabolic-marching method for the prediction of three-dimensional viscous incompressible turbomachinery flows, $\mathrm{PhD}$ thesis, Department of Aerospace Engineering, The Pennsylvania State University

Kirtley K R, Lakshminarayana B $1988 \mathrm{~J}$. Turbomachinery 110: 549-556

Kwak D, Chang J L C, Shanks, Chakravarthy S R 1986 AIAA J. 24: 390-398

Lakshminarayana B 1986 AIAA J. 24: 1900-1917 
Lakshminarayana B, Popovski P 1987 J. Turhomachinery 109: 91-98

McNally W D, Sockol P 1981 J. Fluids Eng. 107: 6-30

Peterson C R 1958 Boundary layer on an airfoil in a cascade, Mrt Gas Turbine Laboratory, Rep. No. 49, December

Popovski P, Lakshminarayana B 1986 AlAA J. 24: 1337-1345

Pouagare M P 1984 Numerical and experimental investigation of turbomachinery rotor flow fields, $\mathrm{PhD}$ thesis, Department of Aerospace Engineering, The Pennsylvania State University

Pouagare M P, Lakshminarayana B 1986 J. Comput. Phys. 64: 389-415

Pouagare M, Galmes J, Lakshminarayana B 1985 ASME J. Eng. Power 107: 364-373

Rodi W 1976 Z. Angew. Math. Mech. 56: 219-221

Rubin S 1980 First Symposium on Numerical and Physical Aspects of Aerodynamic Flows (Long Beach, CA: Springer-Verlag) p. 171

Taylor A M K P, Whitelaw J H, Yianneskis M 1981 Measurement of laminar and turbulent flow in a curved duct with thin inlet boundary layers, NASA CR-3367

Taylor A M K P et al 1982 Developing flow in S-shaped ducts, I-square cross-section duct, NASA CR-3550

Warfield M 1987 A zonal equation method for three-dimensional locally elliptic laminar and turbulent flows, PhD thesis, Department of Aerospace Engineering, The Pennsylvania State University

Warfield M J, Lakshminarayana B 1987 AIAA J. 25: 957-964

Warfield M J, Lakshminarayana B 1987 Calculation of a three-dimensional locally elliptic flow with a zonal equation method, AIAA-87-1141-CP, Proceedings of 8th Computational Fluid Dynamics Conference (Washington, DC: AIAA)

White F 1974 Viscous fluid flow (New Yc k: McGraw-Hill) 